\title{
Solving a Mixed Integer Linear Program Approximation of the Toll Design Problem Using Constraint Generation within a Branch and Cut Algorithm
}

Joakim Ekström, Clas Rydergren and Agachai Sumalee

\author{
Linköping University Post Print
}

\section{Tweet}

N.B.: When citing this work, cite the original article.

Original Publication:

Joakim Ekström, Clas Rydergren and Agachai Sumalee, Solving a Mixed Integer Linear Program Approximation of the Toll Design Problem Using Constraint Generation within a Branch and Cut Algorithm, 2014, Transportmetrica A: Transport Science, (10), 9, 791-819.

http://dx.doi.org/10.1080/23249935.2013.813988

Copyright: (C) 2013 Hong Kong Society for Transportation Studies Limited http://www.tandfonline.com/

Postprint available at: Linköping University Electronic Press http://urn.kb.se/resolve?urn=urn:nbn:se:liu:diva-76642 


\title{
Solving a Mixed Integer Linear Program Approximation of the Toll Design Problem Using Constraint Generation within a Branch and Cut Algorithm
}

\author{
Joakim Ekström $^{\text {*** }}$, Clas Rydergren ${ }^{a}$ and Agachai Sumalee ${ }^{b}$ \\ ${ }^{a}$ Department of Science and Technology, \\ Linköping University, Norrköping, Sweden \\ ${ }^{b}$ Department of Civil and Structural Engineering, \\ The Hong Kong Polytechnic University, Hong Kong, China
}

\begin{abstract}
This paper addresses the global optimality of the toll design problem (TDP) by formulating a mixed integer linear program (MILP) approximation. In the TDP, the objective is to maximize the social surplus by adjusting toll locations and levels in a road traffic network. The resulting optimisation problem can be formulated as a mathematical program with equilibrium constraints (MPEC).

A MILP is obtained by piecewise linear approximation of the non-linear functions in the TDP, and we present a domain reduction scheme to reduce the error introduced by these approximations. Previous approaches for solving the MILP approximation have been relying on a large number of MILPs to be solved iteratively within a cutting constraint algorithm (CCA). This paper instead focuses on the development of a solution algorithm for solving the MILP approximation in which the CCA is integrated within a branch and cut algorithm, which only requires one MILP to be solved.
\end{abstract}

\section{Introduction}

In the toll design problem (TDP) we search for the social welfare maximizing toll locations and corresponding toll levels in a road traffic network. The road users are assumed to be distributed over the links in the traffic network according to a user equilibrium (UE), in which the relationship between travel demand and travel cost is given by a travel demand function. The TDP can be formulated as a bilevel program in which the social surplus is maximized on the upper level by adjusting toll locations and levels, and on the lower level the UE problem is solved to obtain the traffic flow distribution in the network for a given congestion pricing scheme. Formulating the UE as a variational inequality (VI) problem, the bilevel program can be stated as a mathematical program with equilibrium constraints (MPEC). Independent of if the bilevel formulation or the MPEC formulation of the TDP is considered, the resulting optimisation program is both non-convex and non-smooth, and therefore difficult to solve for a global optimum. For a

${ }^{*}$ Corresponding author. Email: joaek@itn.liu.se 
thorough review on optimisation approaches used for solving the TDP see Tsekeris and Voß (2009).

If all links are tollable, without any restrictions on the toll levels and no cost is associated with collecting the tolls, the TDP is convex, and its optimal solution is obtained by letting the road users pay for their external negative effects (Beckmann et al., 1956). Such a pricing scheme is usually referred to as marginal social cost pricing (MSCP). The traffic link flow and the travel demand solution to the UE associated with MSCP is commonly denoted as system optimal (SO). When there are restrictions on toll locations and/or levels and when there is a cost associated with collecting the tolls, the SO link flows and travel demands can be used for constructing an optimistic estimation of the optimal objective function value to the TDP. Such an optimistic estimation can, however, be expected to be far from the true optimal objective function value.

To solve the TDP several heuristic approaches have been suggested. Verhoef (2002) introduces location indices, computable for each link, which are used in a heuristic to find toll locations which yield a high benefit. This approach has however been shown, in Shepherd and Sumalee (2004), to overlook toll locations which yield a high benefit if tolled simultaneously. In both Yang and Zhang (2003) and Shepherd and Sumalee (2004) genetic algorithms are used to solve the TDP, and in Sumalee (2007) a genetic algorithm is presented with the capability to include further constraints on the cordon design. A smoothing technique is applied in Ekström et al. (2009), which replaces the discrete toll location variables by continuous functions of toll levels, so that the problem can be solved by a local optimal approach. While these methods have shown to be able to find good feasible solutions to the TDP, there are no methods available which can provide better optimistic estimations of the global optimal objective function value, compared with what can be estimated with the help of SO traffic link flows and travel demands. Additionally, recent works by Zhang et al. (2011) and Koh et al. (2012) address optimal congestion pricing under competition between cities or regions.

In Ekström et al. (2012) the TDP is tackled by formulating a mixed integer linear program (MILP), to approximate the non-linear functions in the MPEC formulation of the TDP. Similar approach have also been used in Wang and Lo (2010), Luathep et al. (2011) and Farvaresh and Sepehri (2011) and for network design problems (NDP), and in Zhang and van Wee (2012) for a toll design problem related to improving network capacity. While the approaches in Ekström et al. (2012) and Luathep et al. (2011) are based on the VI formulation of the UE, the approches in Wang and Lo (2010), Farvaresh and Sepehri (2011) and Zhang and van Wee (2012) are based on the complementarity constraint formulation of the UE.

In both Ekström et al. (2012) and Luathep et al. (2011) a cutting constraint algorithm (CCA) (see Marcotte, 1983; Lawphongpanich and Hearn, 2004) is applied to solve the MILP, to deal with the VI constraints. This approach, however, requires one mixed integer linear program to be solved in each iteration of the CCA and does not make use of the fact that the only difference between two consecutive iterations in the CCA is one VI constraint. In Ekström et al. (2012), the TDP is formulated with fixed travel demand, while the travel demand is assumed to be elastic in most practical applications of congestion pricing. This paper therefore extends the MILP approximation of the TDP, from Ekström et al. (2012), to traffic networks with elastic travel demand. The extension requires piecewise linear approximation of the functions related to travel 
demand. While such an extension is straightforward, it increases the number of variables and constraints in the MILP approximation, and the number of iterations with the CCA when solving the MILP. Applying the CCA, from Ekström et al. (2012), to this extension has shown to only be practical for small networks. Although the CCA is used for the Sioux Falls network (87 links and 30 OD pairs) in Ekström et al. (2012) it is not able to produce any feasible solution when elastic demand is introduced. In this paper we present a solution method for solving the MILP in which the CCA is integrated in a branch and cut algorithm, requiring only one MILP to be solved. The solution method presented in this paper is not limited to the TDP and can also be applied to e.g. the NDP presented in Luathep et al. (2011).

For solving mixed integer non-convex programs, Floudas (2000) and Tawarmalani and Sahinidis (2002) suggest convexification schemes. The MILP approximation presented in this paper can be regarded as a special case of such a convexification approach, but in which all non-linear functions are approximated by piecewise linear ones, and in which the piecewise linearization scheme is predetermined and limited to a fixed number of linear segments. In both Floudas (2000) and Tawarmalani and Sahinidis (2002) domain reduction is suggested to obtain tighter approximations. Domain reduction strategies restrict the feasible space by imposing variable bounds. The bounds will not interfere with the optimal solution, but can reduce the error introduced by the convexification. Domain reduction can in itself be computationally demanding when working with non-convex programs. In this paper two linear programs are formulated and solved for each link to obtain tighter bounds on the link flows. The domain reduction, applied in this paper, can be considered as a preprocessing of the MILP approximation, to reduce the error introduced by the piecewise linear approximation.

In Ekström et al. (2012) an approximation scheme based on a large number of link flow segments is used, resulting in a MILP with a large number of binary variables. In the MILP formulation presented in this paper, the SO and the untolled link flow solution is used for determining a link flow segmentation with a reduced number of link flow segments.

The remainder of this paper is outlined as follows. In Section 2, the toll design problem is formulated and in Section 3 its MILP approximation is presented. The solution algorithm is developed in Section 4, and in Section 5 the preprocessing scheme, based on domain reduction, is presented, and the choice of break points for the link flow segmentation is discussed. In Section 6 we provide numerical results for the Sioux Falls network and in Section 7 we discuss our findings and make some concluding remarks. Throughout this paper a number of optimization problems are formulated and discussed. In order to help the reader to keep track of these optimization problems, they are summarised in Appendix A.

\section{Problem formulation}

\subsection{The user equilibrium problem}

The traffic network is modelled by a set of links $\mathcal{A}$, and a set of origin destination (OD) pairs $\mathcal{I}$. Let $v$ be the vector of link flows, with $v_{a}$ denoting the flow on link $a$, and let $q$ be the demand vector, with $q_{i}$ equal to the travel demand by car in OD pair $i$. 
Furthermore let $\Omega$ be the set of feasible link flow and demand vectors

$$
\Omega=\left\{(q, v): v=\sum_{k \in \mathcal{I}} x^{k}, B x^{i}=b_{i} q_{i}, q_{i} \geq 0, x^{i} \geq 0, i \in \mathcal{I}, v \leq v^{\max }\right\},
$$

where $x^{i}$ is a vector of link flows, disaggregated by OD pair $i \in \mathcal{I}$, and $B$ is the linknode incidence matrix for the network. The vector $b_{i}$ has length equal to the number of nodes, and defines the origin and destination nodes in OD pair $i$, with the element at the position of the origin node equal to -1 , that of the destination node equal to 1 , and the remaining elements equal to 0 . In $\Omega, v^{\max }$ is the vector of maximum link flows, and the introduction of $v^{\max }$ will ensure that $\Omega$ is a bounded polyhedron. The link travel time is assumed to be a convex increasing function $t_{a}$ of $v_{a}$, and the cost of travelling on link a is made up of both the link travel time, $t_{a}\left(v_{a}\right)$, and the link toll, $\tau_{a}$. The link travel cost is expressed as $c_{a}\left(\tau_{a}, v_{a}\right)=\alpha t_{a}\left(v_{a}\right)+\tau_{a}$, where $\alpha$ is the value of time.

Under the assumption that the road users have perfect information of travel costs in the network and choose the routes which minimizes their individual travel cost, the distribution of the road users on the links within the network corresponds to a user equilibrium (UE). For the UE with elastic demand it is further assumed that a road user only makes a trip by car if this is beneficial. The relationship between travel cost and demand, in each OD pair $i$, is then given by the inverse travel demand function $D_{i}^{-1}\left(q_{i}\right)$, which is assumed to be a convex decreasing function of travel demand $q_{i}$, and gives the travel cost in OD pair $i$, at flow $q_{i}$.

The user equilibrium link flows and travel demands can, for a given toll vector $(\tau)$, be obtained by solving the following convex program (Sheffi, 1985)

$$
\text { P-UE: } \min _{(q, v) \in \Omega} G(q, v)=\sum_{a \in \mathcal{A}} \int_{0}^{v_{a}} c_{a}\left(\tau_{a}, u\right) d u-\sum_{i \in \mathcal{I}} \int_{0}^{q_{i}} D_{i}^{-1}(w) d w .
$$

Note that for P-UE to be a convex program, $t_{a}\left(v_{a}\right)$ and $D_{i}^{-1}\left(q_{i}\right)$ need to be monotonically increasing and decreasing functions respectively. The assumption of convexity for $t_{a}\left(v_{a}\right)$ and $D_{i}^{-1}\left(q_{i}\right)$ is a further restriction needed for the MILP approximation presented in the next section.

Since there is a positive cost associated with travelling on a link, there will be no route flows in the optimal solution to P-UE which include cycles. Removing the bounds $\left(v^{\max }\right)$ from $\Omega$ would give a polyhedral set, and, for P-UE, extreme points in this set are related to routes with no cycles through the network, and extreme rays are related to routes with cycles through the network. Thus the optimal solution to P-UE will always be one of the extreme points, which allow us to introduce $v^{\max }$ in $\Omega$ without removing an optimal solution (if $v^{\max }$ is set sufficiently high).

A VI formulation is adopted to describe the UE with elastic demand (Dafermos, 1980). The VI is defined against all feasible demand-link flow vectors $(\tilde{q}, \tilde{v}) \in \Omega$. Since $\Omega$ is a bounded polyhedron with a finite number, $\mathrm{S}$, of extreme points, $\left(\hat{q}^{s}, \hat{v}^{s}\right), s=1, \ldots, S$, the VI can be formulated as

$$
\sum_{a \in \mathcal{A}} c_{a}\left(\tau_{a}, v_{a}\right)\left(v_{a}-\hat{v}_{a}^{s}\right)-\sum_{i \in \mathcal{I}} D_{i}^{-1}\left(q_{i}\right)\left(q_{i}-\hat{q}_{i}^{s}\right) \leq 0, \quad s=1, \ldots, S .
$$

For proof see Lawphongpanich and Hearn (2004). 


\subsection{Formulating the toll design problem}

Let $y$ be the toll location variables, with $y_{a}$ taking on the value of 1 if a toll is located on link $a$, and the value of 0 otherwise, and let $\tau_{a}^{\max }$ be an upper bound on the toll level at link $a$. The set of feasible toll locations and levels can then be formulated as

$$
\left\{\tau, y \mid y_{a} \in\{0,1\}, 0 \leq \tau_{a} \leq y_{a} \tau_{a}^{\max }, a \in \mathcal{A}\right\} .
$$

The social surplus measure is used for evaluating a congestion pricing scheme, given by the toll locations $y$ and toll levels $\tau$, resulting in user equilibrium link flows $v$ and demands $q$. The social surplus is the sum of the user surplus and operator surplus, and the user surplus is given by

$$
U S(\tau, q, v)=\sum_{i \in \mathcal{I}} \int_{0}^{q_{i}} D_{i}^{-1}(w) d w-\sum_{a \in \mathcal{A}}\left(\alpha t_{a}\left(v_{a}\right)+\tau_{a}\right) v_{a} .
$$

The first sum in the user surplus is the user benefits given by the Marshallian measure (see e.g. Zerbe and Dively, 1994), the second sum is the total user costs. The operator surplus is given by

$$
O S(\tau, v, y)=\sum_{a \in \mathcal{A}}\left(\tau_{a} v_{a}-\chi_{a} y_{a}\right),
$$

where parameter $\chi_{a}$ is the fixed operator costs associated with the location of a toll on link $a$.

Maximizing the social surplus under the constraint that the road users are distributed according to a UE with elastic demand can be formulated as a MPEC, in which the VI formulation of the UE is included as constraints. Since the collected tolls are included in both the user surplus, as a cost, and in the operator surplus, as revenue, they appear twice in the social surplus measure with different signs, and are therefore cancelled out in the objective function of the TDP. The TDP is expressed as the following minimization program

$$
\begin{aligned}
& \text { P-TDP: } \min _{\tau, q, v, y} F_{\mathrm{TDP}}(\tau, q, v, y)=-\sum_{i \in \mathcal{I}} \int_{0}^{q_{i}} D_{i}^{-1}(w) d w+\sum_{a \in \mathcal{A}} \alpha t_{a}\left(v_{a}\right) v_{a}+\sum_{a \in \mathcal{A}} \chi_{a} y_{a} \\
& \text { subject to } \sum_{a \in \mathcal{A}} c_{a}\left(\tau_{a}, v_{a}\right)\left(v_{a}-\hat{v}_{a}^{s}\right)-\sum_{i \in \mathcal{I}} D_{i}^{-1}\left(q_{i}\right)\left(q_{i}-\hat{q}_{i}^{s}\right) \leq 0, \quad s=1, \ldots, S \\
& 0 \leq \tau_{a} \leq y_{a} \tau_{a}^{\max }, \quad a \in \mathcal{A} \\
& y_{a} \in\{0,1\} \text {, } \\
& a \in \mathcal{A} \\
& (q, v) \in \Omega \text {. } \\
& v_{a}^{L} \leq v_{a} \leq v_{a}^{U},
\end{aligned}
$$

Minimizing the objective of P-TDP will maximize the social surplus. Let, for link $a$, $v_{a}^{L}$ be a lower bound and $v_{a}^{U}$ an upper bound on the link flow, which are assumed to be set, for every link, so that any feasible flow in $\Omega$ satisfies constraint (3e). Constraint (3e) is redundant and only included in P-TDP for the domain reduction presented in Section 5.1. For fixed value on the $y$-variables, the problem becomes that of finding optimal toll levels given a predetermined set of tollable links. 


\section{Piecewise linear approximation of the toll design prob- lem}

In P-TDP non-convexity is introduced by the integer variables related to toll locations $y_{a}$, and by $-t_{a}\left(v_{a}\right)$ and $\tau_{a} v_{a}$ in the VI constraints (3a). The convexification approach from Floudas (2000) will approximate P-TDP by a convex program. To improve the convex approximation Floudas (2000) adopts a branch and bound framework, in which branching is either done on continuous variables, to improve the accuracy of the approximation of non-convex functions, or on integer variables, to improve integer feasibility. Branching on continuous variables will in practice divide the link flow space into several segments, and for each segment the non-convex functions will be approximated by linear ones. This approach will require numerous convex programs to be solved. Our approach is instead to use piecewise linear functions to approximate all non-linear functions (even the convex ones), and use a predetermined segmentation of the link flow space, resulting in a mixed integer linear program (MILP). The MILP can then be solved by standard branch and cut (or branch and bound) methods, and will contain two sets of integer variables to branch upon; one set which is related to toll locations (compare to branching on integer variables in Floudas, 2000), and the other which is related to the link flow segmentation (compare to branching on continuous variables in Floudas, 2000). By formulating a MILP it is also possible to take advantage of developed branch and

cut methods, which have been integrated in commercially available solvers, e.g. CPLEX (IBM, 2010) and Gurobi (Gurobi Optimization, 2012).

Rewriting the VI constraints and the objective function, P-TDP can be expressed as

$$
\begin{array}{lr}
\min _{\tau, q, v, y, W} F_{\mathrm{TDP}}(W)=W & \\
\text { subject to } \quad W \geq-\sum_{i \in \mathcal{I}} \int_{0}^{q_{i}} D_{i}^{-1}(w) d w+\sum_{a \in \mathcal{A}} \alpha t_{a}\left(v_{a}\right) v_{a}+\sum_{a \in \mathcal{A}} \chi_{a} y_{a} \\
\sum_{a \in \mathcal{A}}\left(\alpha t_{a}\left(v_{a}\right) v_{a}+\tau_{a} v_{a}-\left(\alpha t_{a}\left(v_{a}\right)+\tau_{a}\right) \hat{v}_{a}^{s}\right)- & \\
\sum_{i \in \mathcal{I}}\left(D_{i}^{-1}\left(q_{i}\right) q_{i}-D_{i}^{-1}\left(q_{i}\right) \hat{q}_{i}^{s}\right) \leq 0, & s=1, \ldots, S \\
v_{a}^{L} \leq v_{a} \leq v_{a}^{U}, & a \in \mathcal{A} \\
0 \leq \tau_{a} \leq y_{a} \tau_{a}^{\text {max }}, & a \in \mathcal{A} \\
y_{a} \in\{0,1\}, & a \in \mathcal{A} \\
(v, q) \in \Omega . &
\end{array}
$$

Since P-TDP is a minimization problem, the variable $W$ and constraint (4a) can be used for expressing the objective function. Except for constraints (4a) and (4b), P-TDP only includes linear terms. The non-linear terms are $t_{a}\left(v_{a}\right) v_{a},-t_{a}\left(v_{a}\right), \tau_{a} v_{a}, D_{i}^{-1}\left(q_{i}\right)$, $-D_{i}^{-1}\left(q_{i}\right) q_{i}$ and $-\int_{0}^{q_{i}} D_{i}^{-1}(w) d w$. Underestimating the right hand side of (4a) and the left hand side of $(4 \mathrm{~b})$, clearly gives a relaxation of the feasible area in P-TDP. By using piecewise linear terms to underestimate $-t_{a}\left(v_{a}\right), t_{a}\left(v_{a}\right) v_{a}, \tau_{a} v_{a}, D_{i}^{-1}\left(q_{i}\right),-D_{i}^{-1}\left(q_{i}\right) q_{i}$ and $-\int_{0}^{q_{i}} D_{i}^{-1}(w) d w$, a MILP can be formulated as an outer approximation of P-TDP. 
Since the MILP approximation will be a relaxation of P-TDP, it will have an optimal objective function value which underestimates the objective function value of P-TDP.

The convex terms $t_{a}\left(v_{a}\right) v_{a}, D_{i}^{-1}\left(q_{i}\right),-D_{i}^{-1}\left(q_{i}\right) q_{i}$ and $-\int_{0}^{q_{i}} D_{i}^{-1}(w) d w$ will be approximated using first-order Taylor approximations. First-order Taylor approximations will, for each function, introduce one additional constraint for each point in which the function is approximated. Taylor approximations can, however, only be used for underestimating the convex functions, and for both the bilinear term $\tau_{a} v_{a}$ and the concave term $-t_{a}\left(v_{a}\right)$ the link flow space needs to be segmented. For each link flow segment a linear (or piecewise linear for the case of the bilinear term) under estimation can then be constructed. The segmentation of link flow space requires the introduction of both additional constraints and binary variables.

In the remainder of this section the piecewise linearization of the non-linear functions are presented, and the resulting MILP formulated. Also, the lower bound estimation, given by the MILP approximation, of the optimal objective function value to P-TDP is compared with what can be achieved using the SO link flow and demand solution.

\subsection{Piecewise linear approximation of convex functions}

Since P-TDP is a minimization problem and the inequalities, which involves non-linear terms, can be expressed in the form $f(x) \leq 0$, the piecewise linear approximations of the convex terms can be formulated by a set of linear inequalities for each term. The assumption of convexity of $t_{a}\left(v_{a}\right) v_{a}$ holds for commonly used travel time functions, e.g. the BPR-function (Bureau of Public Roads, 1964). For the functions related to travel demand, the commonly used linear travel demand function and the power function (with constant elasticity) satisfy the assumption of convexity.

Let $K$ be the number of points in which the function $t_{a}\left(v_{a}\right) v_{a}$ is approximated by first order Taylor approximations, and $T_{k}^{a}$ the link flow at each such point $(k \in 1, \ldots, K)$. The piecewise linear approximation of the total link travel time, $\bar{t}_{a}$, can then be expressed as

$$
\bar{t}_{a} \geq\left. v_{a} \frac{\partial}{\partial v_{a}}\left(t_{a}\left(v_{a}\right) v_{a}\right)\right|_{T_{k}^{a}}+T_{k}^{a}\left(t_{a}\left(T_{k}^{a}\right)-\left.\frac{\partial}{\partial v_{a}}\left(t_{a}\left(v_{a}\right) v_{a}\right)\right|_{T_{k}^{a}}\right), \quad k \in 1, \ldots, K .
$$

Given that the piecewise linearization of $D_{i}^{-1}\left(q_{i}\right),-D_{i}^{-1}\left(q_{i}\right) q_{i}$ and $-\int_{0}^{q_{i}} D_{i}^{-1}(w) d w$ are specified at the $M$ points in demand-space, $Q_{m}^{i}, m \in 1, \ldots, M$, their piecewise linear approximation, $d_{i}, \delta_{i}$ and $\Delta_{i}$ respectively, are given by

$$
\begin{gathered}
D_{i}^{-1}\left(q_{i}\right): d_{i} \geq\left. q_{i} \frac{\partial D_{i}^{-1}\left(q_{i}\right)}{\partial q_{i}}\right|_{Q_{m}^{i}}+D_{i}^{-1}\left(Q_{m}^{i}\right)-\left.Q_{m}^{i} \frac{\partial D_{i}^{-1}\left(q_{i}\right)}{\partial q_{i}}\right|_{Q_{m}^{i}}, m \in 1, \ldots, M \quad \text { (6a) } \\
-D_{i}^{-1}\left(q_{i}\right) q_{i}: \delta_{i} \geq\left.\left(Q_{m}^{i}-q_{i}\right) Q_{m}^{i} \frac{\partial D_{i}^{-1}\left(q_{i}\right)}{\partial q_{i}}\right|_{Q_{m}^{i}}-q_{i} D^{-1}\left(Q_{m}^{i}\right), m \in 1, \ldots, M \quad \text { (6b) } \\
-\int_{0}^{q_{i}} D_{i}^{-1}(w) d w: \Delta_{i} \geq-q_{i} D_{i}^{-1}\left(Q_{m}^{i}\right)-\int_{0}^{Q_{m}^{i}} D_{i}^{-1}(w) d w+Q_{m}^{i} D_{i}^{-1}\left(Q_{m}^{i}\right), m \in 1, \ldots, M .
\end{gathered}
$$

For (6b) and (6c), $Q_{m}^{i}$ have been inserted into the Taylor approximations. 


\subsection{Piecewise linear approximation of non-convex functions}

The approximation of the negative of the travel time function, $-t_{a}\left(v_{a}\right)$, and of the link toll revenue function, $\tau_{a} v_{a}$, require segmentation of the link flow space. For each link flow segment, the linear function which underestimates $-t_{a}\left(v_{a}\right)$, and the convex envelope (Al-Khayyal and Falk, 1983) which underestimates $\tau_{a} v_{a}$ are specified. Let $P$ be the number of linear segments defined by $P+1$ link flow break points. $J_{a, p}$ is the flow at the $\mathrm{p} t h$ break point, and $J_{a, 0}$ will correspond to $v_{a}^{L}$ and $J_{a, P}$ to $v_{a}^{U}$. We define an active link flow segment as a segment with link flow, $v_{a}$, in the interval $\left[J_{a, p} J_{a, p+1}\right]$.

To determine which link flow segment is active at flow $v_{a}$ requires introduction of additional binary variables. Segmenting the link flow space into $n$ segments requires $n-1$ additional binary variables. To reduce the number of binary variables in the MILP, the same segmentation of link flow space will be used both for approximating $-t_{a}\left(v_{a}\right)$ and $\tau_{a} v_{a}$. Increasing the number of segments can increase the accuracy of the approximation, but at the same time the number of binary variables in the MILP will increase. The choice of break points for the link flow segments is further discussed in Section 5 .

There are standard formulations used for treating non-convex piecewise linear functions, and the properties of the formulation presented here are further discussed in Padberg (2000) and Wicaksono and Karimi (2008). The general idea can, however, be traced back to Dantzig (1963). Common for these formulations is the necessity to identify which link flow segment is active.

Let, for each link $a$, the binary variable $\omega_{a, p}$ be equal to 1 if $v_{a} \geq J_{a, p}$ and 0 otherwise, with $p \in 1, \ldots, P-1$. For each link flow segment $\rho_{p, a}$ is the partial link flow corresponding to the flow within each segment, and the total flow $v_{a}$ is the sum of all partial link flows. If $\omega_{a, p}=1$ then $\rho_{a, p}=J_{a, p}-J_{a, p-1}$, and if $\omega_{a, p}=0$ then $\rho_{a, p} \leq J_{a, p}-J_{a, p-1}$ and $\rho_{a, p+1}=0$. Another way to express this is to say that before segment $p+1$ can have a positive partial flow $\rho_{a, p+1} \geq 0$ the $\mathrm{p}$ th segment must have a partial flow $\rho_{a, p}=J_{a, p}-J_{a, p-1}$. We describe these rules by the following set of constraints (Ekström et al., 2012) for each link $a \in \mathcal{A}$ :

$$
\begin{array}{rlrl}
v_{a} & =v_{a}^{L}+\sum_{p=1}^{P} \rho_{a, p} & & \\
\rho_{a, 1} & \leq J_{a, 1}-J_{a, 0} & & \\
\rho_{a, p} & \geq\left(J_{a, p}-J_{a, p-1}\right) \omega_{a, p}, & & p=1, \ldots, P-1 \\
\rho_{a, p+1} & \leq\left(J_{a, p+1}-J_{a, p}\right) \omega_{a, p}, & p=1, \ldots, P-1 \\
\rho_{a, p} \geq 0, & p=1, \ldots, P \\
\omega_{a, p} & \in\{0,1\}, & p=1, \ldots, P-1 .
\end{array}
$$

For the interval $\left[J_{a, p-1}, J_{a, p}\right]$, the underestimation of $-t_{a}\left(v_{a}\right)$ is given by the linear function connecting $\left(J_{a, p-1},-t_{a}\left(J_{a, p-1}\right)\right)$ with $\left(J_{a, p},-t_{a}\left(J_{a, p}\right)\right)$. Note that $p=0$ corresponds to the lower bound, $v_{a}^{L}$, of the link flow on link $a$. Figure 1 illustrates the principle of the piecewise linear approximation of $-t_{a}\left(v_{a}\right)$ for two linear segments.

The piecewise linear underestimation of $-t_{a}\left(v_{a}\right)$ is given by the variable $\theta_{a}$ (Ekström 
et al., 2012)

$$
\theta_{a}=-t_{a}\left(J_{a, 0}\right)-\sum_{p=1}^{P} \frac{t_{a}\left(J_{a, p}\right)-t_{a}\left(J_{a, p-1}\right)}{J_{a, p}-J_{a, p-1}} \rho_{a, p} .
$$

The link toll revenue function, $\tau_{a} v_{a}$, is a bilinear term, and its convex envelopes will give an outer approximation which underestimate the function value. Given the lower and upper bounds on the link flow $\left(v_{a}^{L}, v_{a}^{U}\right)$ and toll level $\left(\tau_{a}^{L}, \tau_{a}^{\max }\right)$ for a link $a$, the convex envelope which outer approximates $\tau_{a} v_{a}$ can be expressed as (Al-Khayyal and Falk, 1983)

$$
\begin{gathered}
\sigma_{a} \geq v_{a} \tau_{a}^{\max }+\tau_{a} v^{U}-v_{a}^{U} \tau_{a}^{\max } \\
\sigma_{a} \geq v_{a} \tau_{a}^{L}+\tau_{a} v^{L}-v_{a}^{L} \tau_{a}^{L},
\end{gathered}
$$

where $\sigma_{a}$ is the outer approximation of $\tau_{a} v_{a}$. Using the link flow segmentation, the error introduced by the approximation, $\tau_{a} v_{a}$, can be reduced by formulating different convex envelopes for each link flow segment, with the minimum and maximum link flows in each segment given by $J_{a, p}$ and $J_{a, p+1}$.

In this paper the approach from Wicaksono and Karimi (2008) is adopted for formulating the convex envelopes for each link flow segment. Assuming that $\tau_{a}^{L}=0$, the approximation of $\tau_{a} v_{a}$, for each link $a$, is given by (7a) - (7f) together with

$$
\begin{aligned}
\tau_{a} & =\sum_{p=1}^{P} \gamma_{a, p} \\
\gamma_{a, 1} & \leq \tau_{a}^{\max }\left(1-\omega_{a, 1}\right) \\
\gamma_{a, P} & \leq \tau_{a}^{\max } \omega_{a, P-1} \\
\gamma_{a, p} & \leq \tau_{a}^{\max }\left(\omega_{a, p-1}-\omega_{a, p}\right), \quad p=2 \ldots P-1 \\
\sigma_{a} & \geq \sum_{p=1}^{P} J_{a, p-1} \gamma_{a, p} \\
\sigma_{a} & \geq v_{a} \tau_{a}^{\max }+\sum_{1=p}^{P} J_{a, p} \gamma_{a, p} \\
& -\tau_{a}^{\max }\left(J_{a, 1}\left(1-\omega_{a, 1}\right)+\sum_{p=2}^{P-1} J_{a, p}\left(\omega_{a, p-1}-\omega_{a, p}\right)+J_{a, P} \omega_{a, P-1}\right) \\
\gamma_{a, p} & \geq 0, \quad p=1, \ldots, P .
\end{aligned}
$$

The variable $\gamma_{a, p}$ is introduced for each link and link flow segment, and will be used to construct the convex envelope for the active segment. If $\omega_{a, \tilde{p}-1}=1$ and $\omega_{a, \tilde{p}}=0$, then constraints (9a)-(9d) will give that $\gamma_{a, \tilde{p}}=\tau_{a}$, and for $p<\tilde{p}$ and $p>\tilde{p}, \gamma_{a, p}=0$. In (9e) and (9f), $\gamma_{a, p}$ is then used when multiplying the minimum and the maximum link flow within the active segment with the toll level. In Ekström et al. (2012) it is shown that the set of constraints (9) will result in a convex envelope, which underestimates the link toll revenue, for each link flow segment. 


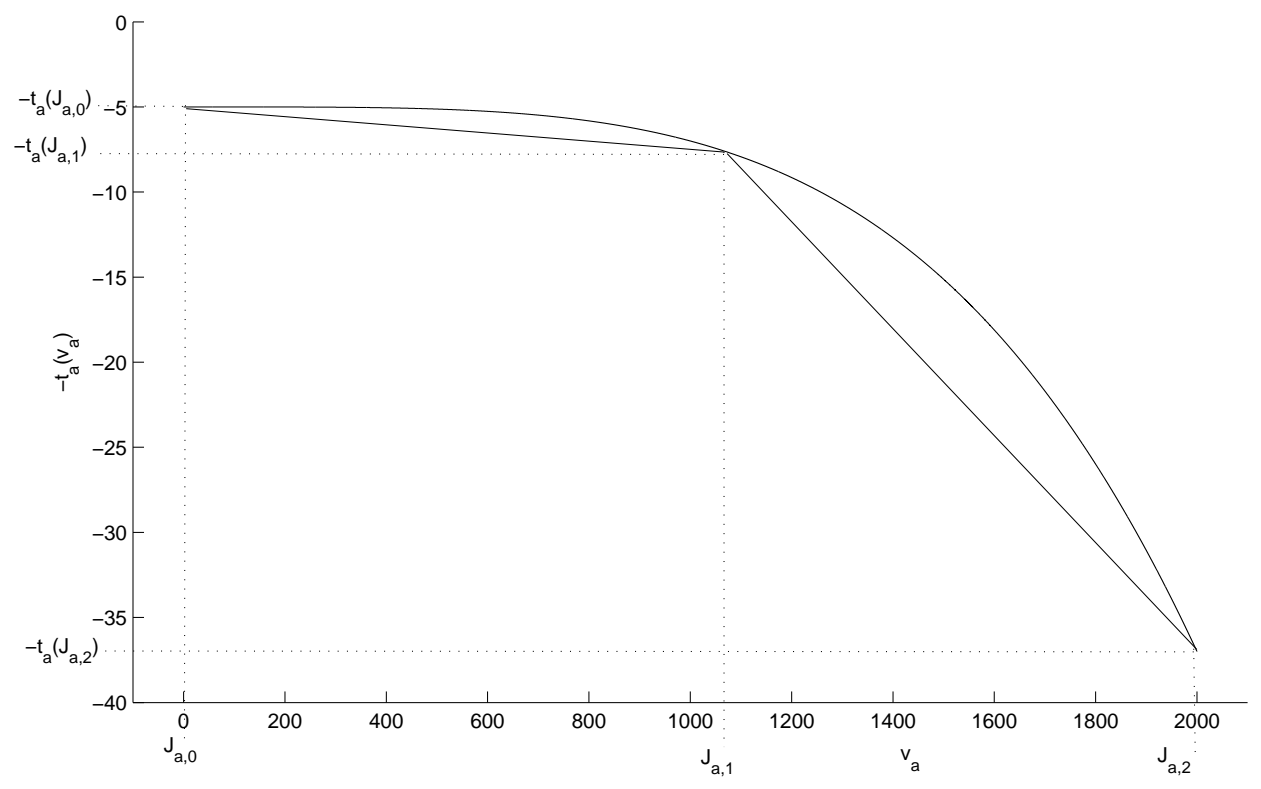

Figure 1: Example of link flow segmentation and the piecewise linear underestimation of $-t_{a}\left(v_{a}\right)$

\subsection{The MILP formulation}

Combining the piecewise linear approximation of the non-linear terms presented in Section 3.1 and 3.2 with P-TDP, a MILP can be formulated. From P-TDP we have the traffic state variables ( $q$ and $v$ ), and the decision variables $(y$ and $\tau$ ). Additionally we have introduced the variables related to approximating the convex functions $(\bar{t}, d$, $\delta$ and $\Delta$ ), the variables related to the segmentation of the link flow space ( $\omega$ and $\rho$ ), the variables related to the approximation of the negative of the travel time function $(\theta)$, and finally the variables related to the approximation of the link toll revenue functions $(\gamma$ and $\sigma)$. For simplicity we will use $x$ to denote the complete set of variables $(\delta, \Delta, \gamma, \theta, \rho, \sigma, \tau, \omega, q, \bar{t}, v, y)$. The MILP has the following structure

$$
\begin{aligned}
& \text { P-MILP: } \min _{x} F_{\mathrm{MILP}}(\Delta, \bar{t}, y)=\sum_{i \in \mathcal{I}} \Delta_{i}+\sum_{a \in \mathcal{A}}\left(\alpha \bar{t}_{a}+\chi_{a} y_{a}\right) \\
& \text { subject to } \sum_{a \in \mathcal{A}}\left(\alpha \bar{t}_{a}+\sigma_{a}+\left(\alpha \theta_{a}-\tau_{a}\right) \hat{v}_{a}^{s}\right)+\sum_{i \in \mathcal{I}}\left(d_{i} \hat{q}_{i}^{s}+\delta_{i}\right) \leq 0, \quad s \in 1, \ldots, S \\
& v_{a}^{L} \leq v_{a} \leq v_{a}^{U} \\
& a \in \mathcal{A} \\
& 0 \leq \tau_{a} \leq y_{a} \tau_{a}^{\max } \\
& a \in \mathcal{A} \\
& y_{a} \in\{0,1\}, \\
& a \in \mathcal{A} \\
& (v, q) \in \Omega \text {, } \\
& \text { and constraints }(5)-(9) \text {, } \\
& a \in \mathcal{A} \text {. }
\end{aligned}
$$


Consider the reformulation of P-TDP in (4). The underestimation of $t_{a}\left(v_{a}\right) v_{a}$ and $-\int_{0}^{q_{i}} D_{i}^{-1}(w) d w$ results in a relaxation of (4a). Also, the underestimation of $t_{a}\left(v_{a}\right) v_{a}$, $-t_{a}\left(v_{a}\right), \tau_{a} v_{a}, D_{i}^{-1}\left(q_{i}\right)$ and $-D_{i}^{-1}\left(q_{i}\right) q_{i}$ in (4b) relaxes constraint (4b). Thus, the optimal solution to P-MILP will give a lower bound estimation of the optimal objective function value to $\mathrm{P}-\mathrm{TDP}$.

\subsection{Lower bound estimation of the optimal objective function value to the toll design problem}

Assuming that we disregard any operator costs, the most efficient distribution of the travelers in the traffic network is achieved with MSCP tolls, resulting in SO link flows and demands. The social surplus associated with SO link flows and demand can be used for underestimating the optimal objective function value of P-TDP. We will now proceed to show that the solution to P-MILP will always produce an underestimation of the optimal objective function value to P-TDP, which is equal to or better than what can be computed by the help of SO link flows and demands. This holds given that the SO link flow and demand solution is included as points of linearization for the convex functions in P-TDP.

SO link flows and demands can be obtained by formulating and solving the convex program (Sheffi, 1985)

$$
\text { P-SO: } \min _{(q, v) \in \Omega} F_{\mathrm{SO}}(q, v)=\sum_{a \in \mathcal{A}} \alpha t_{a}\left(v_{a}\right) v_{a}-\sum_{i \in \mathcal{I}} \int_{0}^{q_{i}} D_{i}^{-1}(w) d w .
$$

Note that the objective in P-SO is equal to the one in P-TDP, except for the operator costs. P-SO is convex and can be solved to its optimal solution $\left(v^{\mathrm{SO}}, q^{\mathrm{SO}}\right)$ with optimal objective function value $F_{\mathrm{SO}}\left(v^{\mathrm{SO}}, q^{\mathrm{SO}}\right) . \quad F_{\mathrm{SO}}\left(v^{\mathrm{SO}}, q^{\mathrm{SO}}\right)$ can be used for underestimating the optimal objective function value to $\mathrm{P}-\mathrm{TDP}$, by recognizing that $F_{\mathrm{SO}}\left(v^{\mathrm{SO}}, q^{\mathrm{SO}}\right)$ is the lowest possible value on the objective function value to P-TDP.

If the non-linear parts of the objective in P-SO are approximated by piecewise linear functions, in the same way as is done for P-TDP, and $\left(v^{\mathrm{SO}}, q^{\mathrm{SO}}\right)$ are included as points of linearization, then the optimal objective function value to the linearized problem will be equal to $F_{\mathrm{SO}}\left(v^{\mathrm{SO}}, q^{\mathrm{SO}}\right)$. This is only valid under the assumption of convex travel time and inverse travel demand functions, and follows from the first-order optimality conditions for convex programs. Now consider a relaxed version of P-MILP, denoted P-MILP-SO, in which the VI constraints have been removed, and in which $\left(v^{\mathrm{SO}}, q^{\mathrm{SO}}\right)$ are included as points of linearization for the convex functions in the objective function. P-MILP-SO will then have an optimal objective function value equal to $F_{\mathrm{SO}}\left(v^{\mathrm{SO}}, q^{\mathrm{SO}}\right)$. Let $x_{\mathrm{MILP}}^{*}$ and $x_{\mathrm{TDP}}^{*}$ be optimal solutions to P-MILP and P-TDP respectively, then the following must hold $F_{\mathrm{SO}}\left(v^{\mathrm{SO}}, q^{\mathrm{SO}}\right) \leq F_{\mathrm{MILP}}\left(x_{\mathrm{MILP}}^{*}\right) \leq F_{\mathrm{TDP}}\left(x_{\mathrm{TDP}}^{*}\right)$. Thus it can be concluded that the underestimation of the optimal objective function value to P-TDP, by the optimal objective function value to P-MILP, in worst case will be equal to the underestimation based on the social surplus associated with SO link flows and demands. 


\section{Solving the MILP}

Several commercially available solvers (e.g. CPLEX and Gurobi) contain branch and cut methods for solving MILPs. Branch and cut methods are commonly described in terms of a binary tree in which each node represents a separation of the original problem, with integrality relaxed. In each node, cutting planes are generated to remove solutions which involve integer variables with fractional values. The main parts of a branch and cut algorithm are branching, bounding, pruning and cutting. Branching on integer variables is done to create new nodes by introducing a separation which makes the current fractional solution infeasible. The separation fixates one binary variable with fractional value to 0 (left branch) or to 1 (right branch). It is common to denote the original problem (in our case P-MILP) as $\mathrm{P}$, and the subproblem at node $n$ as $\mathrm{SP} n$. In each node of the binary tree, one subproblem needs to be solved. A solution to SPn gives (for a minimization problem) a lower bound (LBD) estimation, i.e. an optimistic estimation, of the objective function value which can be obtained in the subtree defined by node $n$ as root node. Any solution to $\mathrm{SP} n$ which is feasible in $\mathrm{P}$ gives an upper bound (UBD) estimation, i.e. a pessimistic estimation, of the optimal objective function value to P. Pruning is done at nodes for which it can be determined that branching will not lead to the optimal solution, i.e. at nodes which the optimal objective function value of $\mathrm{SP} n$ is higher than the UBD, at nodes for which $\mathrm{SP} n$ has an integer solution, and at nodes for which $\mathrm{SP} n$ is infeasible. The solutions are further restricted to solutions which satisfy the integer requirement, by using a cutting plane algorithm in each node. In the root node (node 0) of the tree, SP0 will be a linear program relaxation of $\mathrm{P}$, and in the following subproblems additional integer restrictions are imposed on the binary variables. The same approach can be used without the generation of cutting planes and is then referred to as a branch and bound method. The methodology for solving PMILP, presented in this section, can be applied to both branch and cut and branch and bound methods, but for convenience we will only refer to the branch and cut method. For a more general description of branch and cut and branch and bound methods we refer to Wolsey and Nemhauser (1999).

A branch and cut method can, however, not be directly applied to P-MILP. P-MILP involves a set of linearized VI constraints which requires the complete set of extreme points, $s \in 1, \ldots, S$, to be known. In Ekström et al. (2012) a CCA is used for solving P-MILP, but this approach requires one MILP to be solved in each iteration of the CCA, by repeatedly applying a branch and cut method. Even for the medium sized network used for numerical experiments in this paper, the CCA from Ekström et al. (2012) fails due to the large number of VI constraints which need to be generated. Therefore a CCA will instead be integrated in the branch and cut method, and used for solving each subproblem. This approach can also be applied for solving the NDP in Luathep et al. (2011). 
The subproblem at node $n, \mathrm{SP} n$, can be expressed as

$$
\begin{aligned}
& \text { P-SP: } \min _{x} F_{\mathrm{MILP}}(\Delta, \bar{t}, y)=\sum_{i \in \mathcal{I}} \Delta_{i}+\sum_{a \in \mathcal{A}}\left(\alpha \bar{t}_{a}+\chi_{a} y_{a}\right) \\
& \text { subject to } \sum_{a \in \mathcal{A}}\left(\alpha \bar{t}_{a}+\sigma_{a}+\left(\alpha \theta_{a}-\tau_{a}\right) \hat{v}_{a}^{s}\right)+\sum_{i \in \mathcal{I}}\left(d_{i} \hat{q}_{i}^{s}+\delta_{i}\right) \leq 0, \quad s \in 1, \ldots, S \\
& v_{a}^{L} \leq v_{a} \leq v_{a}^{U}, \quad a \in \mathcal{A} \\
& 0 \leq \tau_{a} \leq y_{a} \tau_{a}^{\max }, \quad a \in \mathcal{A} \\
& (y, \omega) \in \Phi_{n}, \\
& (v, q) \in \Omega \text {, } \\
& \text { and constraints (5), (6), (7a) - (7e), (8) and (9a) }-(9 \mathrm{~g}), \quad a \in \mathcal{A} \text {, }
\end{aligned}
$$

which is a linear program. $\Phi_{n}$ is the additional set of constraints, imposed at node $n$, on the binary variables through the separation process (branching).

Since all VI constraints are not expected to be binding at optimum, the subproblems can be formulated with a reduced number of extreme points, by replacing the complete set of extreme points in P-SP with the set of extreme points $s \in R$, where $R \subseteq\{1, \ldots, S\}$. The subproblem at node $n$, with a reduced set of extreme points $R_{n}$, can then be formulated as

$$
\begin{aligned}
& \mathbf{P}^{-S P} \mathbf{S P}^{\prime}: \min _{x} F_{\mathrm{MILP}}(\Delta, \bar{t}, y)=\sum_{i \in \mathcal{I}} \Delta_{i}+\sum_{a \in \mathcal{A}}\left(\alpha \bar{t}_{a}+\chi_{a} y_{a}\right) \\
& \text { subject to } \sum_{a \in \mathcal{A}}\left(\alpha \bar{t}_{a}+\sigma_{a}+\left(\alpha \theta_{a}-\tau_{a}\right) \hat{v}_{a}^{s}\right)+\sum_{i \in \mathcal{I}}\left(d_{i} \hat{q}_{i}^{s}+\delta_{i}\right) \leq 0, \quad s \in R_{n} \\
& v_{a}^{L} \leq v_{a} \leq v_{a}^{U}, \quad a \in \mathcal{A} \\
& 0 \leq \tau_{a} \leq y_{a} \tau_{a}^{\max }, \quad a \in \mathcal{A} \\
& v_{a}^{L} \leq v_{a} \leq v_{a}^{U}, \quad a \in \mathcal{A} \\
& (y, \omega) \in \Phi_{n} \\
& (v, q) \in \Omega \text {. } \\
& \text { and constraints (5), (6), (7a) - (7e), (8) and (9a) - (9g), } a \in \mathcal{A} \text {. }
\end{aligned}
$$

Let $x^{*}$ denote the optimal solution to $\mathrm{P}_{-\mathrm{SP}^{\prime}}$ at node $n$. The search for an additional VI constraint at node $n$, which is violated by the current solution $x^{*}$, can then be formulated as the linear program

$$
\text { P-LP: } \max _{(\tilde{q}, \tilde{v}) \in \Omega} F_{\mathrm{LP}}(\tilde{q}, \tilde{v})=\sum_{a \in \mathcal{A}}\left(\alpha \bar{t}_{a}^{*}+\sigma_{a}^{*}+\left(\alpha \theta_{a}^{*}-\tau_{a}^{*}\right) \tilde{v}_{a}\right)+\sum_{i \in \mathcal{I}}\left(d_{i}^{*} \tilde{q}_{i}+\delta_{i}^{*}\right)
$$

with optimal solution $\left(\tilde{q}^{*}, \tilde{v}^{*}\right)$. Note that P-LP can be decomposed by origin node, and solved as a shortest path problem for each origin node. If $F_{\mathrm{LP}}\left(\tilde{q}^{*}, \tilde{v}^{*}\right) \leq 0$, then there exists no additional constraint which would make the current solution to $\mathrm{P}-\mathrm{SP}^{\prime}$ infeasible, and the current solution is thus an optimal solution to P-SP. On the other hand, if $F_{\mathrm{LP}}\left(\tilde{q}^{*}, \tilde{v}^{*}\right)>0$, then there exists one extreme point, $\left(\tilde{q}^{*}, \tilde{v}^{*}\right)$, which corresponds to a violated VI constraint. In the CCA, which will be used for solving $\mathrm{P}-\mathrm{SP}, \mathrm{P}-\mathrm{SP}^{\prime}$ and 
P-LP will be solved repeatedly. P-LP will either indicate that the optimal solution to $\mathrm{P}-\mathrm{SP}$ has been reached, or identify a new VI constraint to be added to P-SP'.

Let $x_{\mathrm{SP}}^{*}$ and $x_{\mathrm{SP}^{\prime}}^{*}$ be the optimal solutions to $\mathrm{P}-\mathrm{SP}$ and $\mathrm{P}-\mathrm{SP}^{\prime}$ respectively, then $F_{\mathrm{SP}}\left(x_{\mathrm{SP}^{\prime}}^{*}\right) \leq F_{\mathrm{SP}}\left(x_{\mathrm{SP}}^{*}\right)$. Thus, if the solution to $\mathrm{P}-\mathrm{LP}$ indicates that $\mathrm{P}_{-\mathrm{SP}^{\prime}}$ violates some VI constraint, the current objective function value of $\mathrm{P}_{-} \mathrm{SP}^{\prime}$ will still be a valid lower bound in the branch and cut method. If $x_{\mathrm{SP}^{\prime}}^{*}$ is feasible in P-MILP, with respect to the values on the binary variables $(y$ and $\omega)$ being integer, but not necessarily to the VI constraints, the solution is referred to as an integer feasible solution. In contrast to the case when $x_{\mathrm{SP}^{\prime}}^{*}$ has binary variables with fractional values, which is referred to as a integer infeasible solution.

To generate VI constraints in all nodes until no more VI constraints can be found is time consuming, and time will be spent on generating constraints for subproblems which will not lead to the optimal solution. A control parameter $\epsilon \geq 0$ is therefore introduced to allow the CCA to terminate with a solution which is not feasible with respect to the VI constraints. Premature termination of the CCA will, however, only be allowed if the current solution to $\mathrm{P}-\mathrm{SP}$ is integer infeasible, and for this case no feasible solution to $\mathrm{P}-\mathrm{SP}$ is obtained. The objective function value to $\mathrm{P}_{-} \mathrm{SP}^{\prime}$ is, however, returned to the branch and cut method, where it is used as a lower bound estimation of what can be achieved if proceeding to branch from node $n$. If the solution to P-SP is integer feasible any violated VI constraint is added to ensure that an integer feasible solution obtained within the branch and cut method always is a feasible solution to P-MILP with respect to the VI constraints.

The cutting constraint algorithm for solving the subproblem SPn (SP-CCA) at node $n$ is outlined in Figure 2, and formulated as

\section{SP-CCA:}

Step 0 . Set iteration counter $j:=0$, and let $R_{n}$ be the initial set of extreme points. Set $U B D$ equal to the current upper bound in the branch and cut search tree.

Step 1. Set $j:=j+1$. Solve P-SP , which gives the solution $x^{*}$ and objective function value $F_{\mathrm{SP}}^{j}\left(x^{*}\right)$, and P-LP, which gives the solution $\left(\tilde{q}^{*}, \tilde{v}^{*}\right)$ and objective function value $F_{\mathrm{LP}}\left(\tilde{q}^{*}, \tilde{v}^{*}\right)$. If $F_{\mathrm{SP}}^{j}\left(x^{*}\right) \geq U B D$ continued branching will not lead to the optimal solution to P-MILP, and the algorithm can be terminated. If ${\mathrm{P}-\mathrm{SP}^{\prime}}^{\prime}$ is infeasible P-SP must also be infeasible, and the algorithm can be terminated (the current node can be pruned).

Step 2. If the solution to $\mathrm{P}_{-} \mathrm{SP}^{\prime}$ is integer feasible and $F_{\mathrm{LP}}\left(\tilde{q}^{*}, \tilde{v}^{*}\right) \leq 0$ terminate the algorithm with $x^{*}$ being the optimal solution to P-SP, otherwise continue with Step 3.

Step 3. If the solution to $\mathrm{P}-\mathrm{SP}^{\prime}$ is integer feasible and $F_{\mathrm{LP}}\left(\tilde{q}^{*}, \tilde{v}^{*}\right)>0$ set $R_{n} \cup\left(\tilde{q}^{*}, \tilde{v}^{*}\right)$ and continue with Step 1, otherwise continue to Step 4.

Step 4. If $F_{\mathrm{LP}}\left(\tilde{q}^{*}, \tilde{v}^{*}\right) \leq \epsilon$ terminate the algorithm with $x^{*}$ being the final solution at node $n$, otherwise set $R_{n} \cup\left(\tilde{q}^{*}, \tilde{v}^{*}\right)$, and continue with Step 1 .

SP-CCA will terminate if

(i) there exists no feasible solution to $\mathrm{P}-\mathrm{SP}^{\prime}$ 
(ii) the optimal objective function value to $\mathrm{P}_{-} \mathrm{SP}^{\prime}$ is higher than the currently best found UBD to P-MILP

(iii) the optimal solution to $\mathrm{P}_{-} \mathrm{SP}^{\prime}$ is integer feasible, and the optimal objective function value to $\mathrm{P}$-LP is less than or equal to 0, i.e. the optimal solution to P-SP has been obtained

(iv) the optimal solution to $\mathrm{P}_{-} \mathrm{SP}^{\prime}$ is integer infeasible and the optimal objective function value of P-LP is less than or equal to $\epsilon$

For (i), (ii) and (iii) the current node can be pruned, for (iv) branching will be continued. For (iii) and (iv) it is important to note that SP-CCA will always continue to generate new VI constraints if the optimal solution to ${\mathrm{P}-\mathrm{SP}^{\prime}}^{\prime}$ is integer feasible, and $\mathrm{P}$ LP indicates that some VI constraints are violated. This ensures that any integer feasible solution obtained in one of the branch and cut nodes (after SP-CCA has terminated) is a feasible solution to P-MILP and an upper bound estimation of the optimal objective function value to P-TDP. Using $\epsilon>0$ will not affect the execution of the branch and cut method, in which nodes will only be pruned if they are either infeasible, feasible with respect to the integer variables, or can be determined to not lead to an optimal solution.

In each iteration of SP-CCA one additional extreme point of $\Omega$ will be obtained and the corresponding VI constraint added. The set of extreme points of $\Omega$ is finite and thus the SP-CCA will terminate when $F_{\mathrm{LP}}\left(\tilde{q}^{*}, \tilde{v}^{*}\right) \leq 0$, for an integer feasible solution, when $F_{\mathrm{LP}}\left(\tilde{q}^{*}, \tilde{v}^{*}\right) \leq \epsilon$, for an integer infeasible solution, or when all extreme points of $\Omega$ have been obtained. Thus, SP-CCA will terminate in a finite number of iterations. Setting $\epsilon$ to a low value will lead to a larger number of nodes being pruned earlier in the search, but on the other hand increase the number of iterations within SP-CCA.

Any VI constraint generated at node $n$ is a valid VI constraint at any node in the branch and cut tree. Two main strategies can be used for adding VI constraints; locally or globally. Adding a VI constraint locally, at node $n$, means that the constraint will be added to the subproblem at any node in the subtree which is defined by node $n$ as root node. To add a constraint globally means that the constraint will be included in any future node subproblem solved. While adding constraints globally may lead to larger subproblems, the constraints can, in CPLEX, be added as lazy constraints (IBM, 2010). A lazy constraint will only be included in the subproblem if it will actually restrict the solution, thus by using lazy constraints the VI constraints can be added globally without increasing the size of the subproblems unnecessary. There will, however, be a slight increase in computational time even if lazy constraints are used, since checking if any lazy constraint is violated must be done each time $\mathrm{P}_{-} \mathrm{SP}^{\prime}$ is solved. Adding lazy constraints globally has shown to be the best choice in our implementation, when integrating SP-CCA with the branch and cut method in the commercial solver CPLEX.

The implementation of SP-CCA within CPLEX has been validated on the nine node network from Yildirim and Hearn (2005). With a large enough number of piecewise linear segments, the validation has shown that the global optimal solution to P-TDP is obtained by solving P-MILP. 


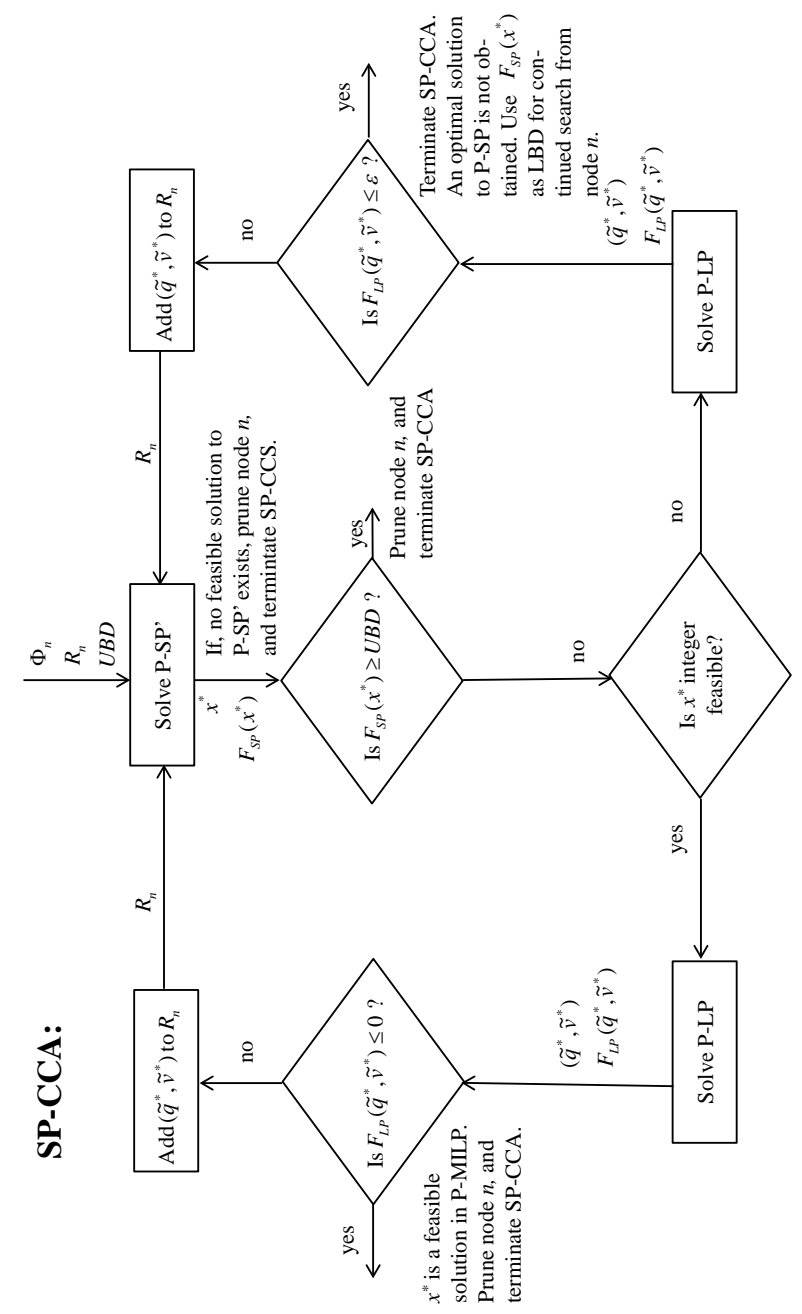

Figure 2: The SP-CCA algorithm for solving P-SP at node $n$

\section{$5 \quad$ Segmenting the link flow space}

\subsection{A preprocessing scheme}

For the non-convex terms $-t_{a}\left(v_{a}\right)$ and $\tau_{a} v_{a}$, the piecewise linear approximation will depend on the link flow segmentation. Each additional segment will, for each link, generate one additional binary variable, and to reduce the size of the MILP it is therefore of interest to keep the number of link flow segments as small as possible. Using only one segment requires no binary variables, and to use two segments requires one. Note that the bounds $v_{a}^{L}$ and $v_{a}^{U}$ will affect the approximation error. If $v_{a}^{L}$ can be increased and $v_{a}^{U}$ decreased the possible error introduced by the approximation is reduced. Figure 3 gives an example of a link flow segmentation with two segments, in which (1) represents the initial bound (with $v_{a}^{L}=J_{a, 0}^{(1)}$ and $v_{a}^{U}=J_{a, 2}^{(1)}$ ) and (2) the updated bounds (with $v_{a}^{L}=J_{a, 0}^{(2)}$ and $\left.v_{a}^{U}=J_{a, 2}^{(2)}\right)$. Changing the value on these bounds may restrict the feasible 
link flows in P-TDP (and P-MILP). This is usually referred to as domain or range reduction (Floudas, 2000). Caprara and Locatelli (2010) describe two different types of domain reductions; feasibility-based domain reductions, in which all link flow solutions will remain feasible; and optimality-based domain reductions, which may remove feasible link flow solutions but without removing the optimal one.

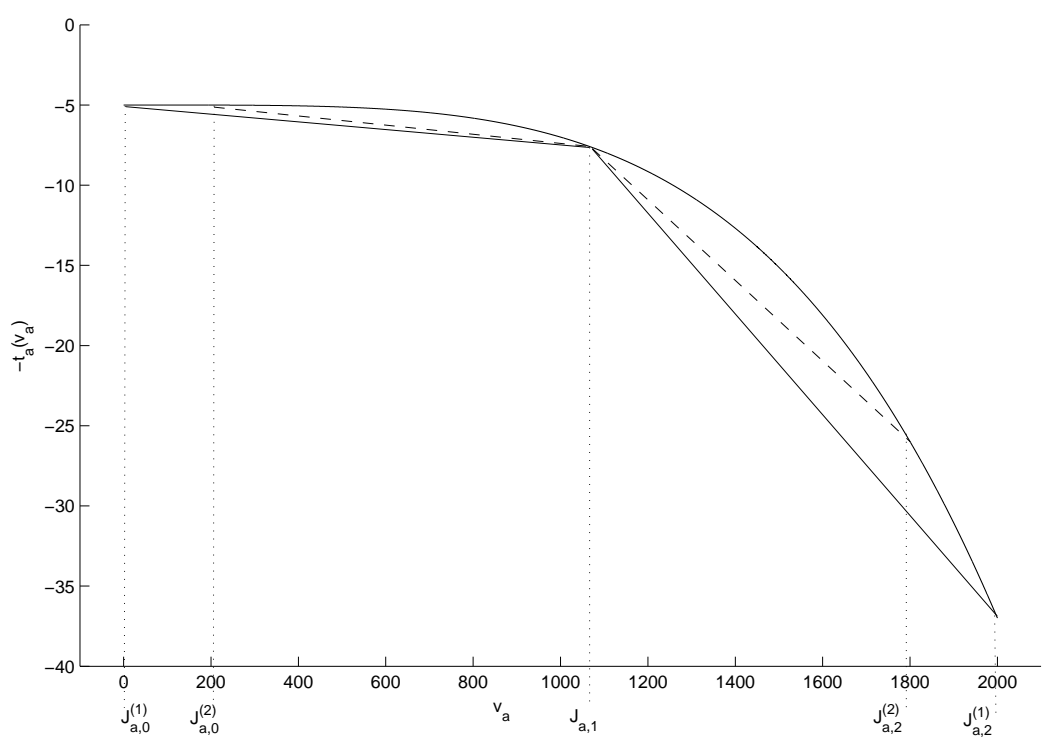

Figure 3: Example of the negative of a travel time function and its piecewise linear underestimation defined by initial bounds (1), and updated bounds (2).

Consider the following program

$$
\begin{array}{cl}
\text { P-MILP-R: } & \min _{\Delta, q, \bar{t}, v} F_{\text {MILP-R }}(\Delta, \bar{t})=\sum_{i \in \mathcal{I}} \Delta_{i}+\sum_{a \in \mathcal{A}} \alpha \bar{t}_{a}+\hat{C} \\
& \text { subject to } \quad v_{a}^{L} \leq v_{a} \leq v_{a}^{U}, \quad a \in \mathcal{A} \\
& (v, q) \in \Omega, \\
& \text { and constraints } \quad(5) \text { and }(6 c),
\end{array}
$$

which is P-MILP, but with the VI constraints removed and the parameter $\hat{C}$ replacing the operator costs in the objective function. The parameter $\hat{C}$ is a lower bound estimation on the operator costs in the optimal solution to P-TDP, and $\hat{C}=0$ is always a valid lower bound. Note that the toll level variables $\tau$ are only included in the VI constraints, and thus neither the toll location nor the toll level variables are included in P-MILP-R.

Let $x^{*}$ and $\hat{x}^{*}$ denote the optimal solution to P-TDP and P-MILP-R respectively, and let $\bar{x}$ be a known feasible solution to P-TDP. Then the following will always hold $F_{\mathrm{TDP}}(\bar{x}) \geq F_{\mathrm{TDP}}\left(x^{*}\right) \geq F_{\mathrm{MILP}-\mathrm{R}}\left(\hat{x}^{*}\right)$.

Let $\Xi$ be the feasible region defined by any $(\Delta, q, \bar{t}, v)$ which satisfy the constraints (10b)-(10d) and the constraint $\sum_{i \in \mathcal{I}} \Delta_{i}+\sum_{a \in \mathcal{A}} \alpha \bar{t}_{a}+\hat{C} \leq \hat{F}_{\mathrm{TDP}}$, with $\hat{F}_{\mathrm{TDP}}:=F_{\mathrm{TDP}}(\bar{x})$. 
Proposition 5.1. a) An optimal solution to P-TDP, $x^{*}$, must satsify $\Xi$.

b) Let $v^{*}$ denote the vector of optimal link flows to P-TDP. Minimizing $v_{b}$ over $\Xi$ gives the link flow $v_{b}^{\prime}$, which will be used for updating the lower bound $v_{b}^{L}:=v_{b}^{\prime}$. For an optimal solution to $P$-TDP,$v_{b}^{*} \geq v^{\prime}{ }_{b}$ will always hold. Maximizing $v_{b}$ over $\Xi$ gives the link flow $v_{b}^{\prime \prime}$ which will be used for updating the upper bound $v_{b}^{U}:=v_{b}^{\prime \prime}$. For an optimal solution to $P-T D P, v_{b}^{*} \leq v_{b}^{\prime \prime}$ will always hold.

Proof. a) It is only the $(\Delta, q, \bar{t}, v)$ elements in $x^{*}$ that are included in P-MILP-R. The feasible area of P-MILP-R is a relaxation of P-TDP. Thus $\left(\Delta^{*}, q^{*}, \bar{t}^{*}, v^{*}\right)$ will satsify (10b)-(10d). Since $\hat{F}_{\mathrm{TDP}} \geq F_{\mathrm{TDP}}\left(x^{*}\right)$ and $\hat{C} \leq \sum_{a \in \mathcal{A}} \chi_{a} y_{a}^{*}, \quad\left(\Delta^{*}, \bar{t}^{*}\right)$ must satsify $\sum_{i \in \mathcal{I}} \Delta_{i}^{*}+\sum_{a \in \mathcal{A}} \alpha \bar{t}_{a}^{*}+\hat{C} \leq \hat{F}_{\mathrm{TDP}}$.

b) An optimal solution to P-TDP will always satisfy $\Xi$, thus minimizing $v_{b}$ will give the lowest flow on link $b$, which could possibly be obtained in an optimal solution to P-TDP. The same holds for maximizing $v_{b}$.

The domain reduction strategy described in Proposition 5.1 is clearly an optimalitybased strategy. If the constraint $\sum_{i \in \mathcal{I}} \Delta_{i}+\sum_{a \in \mathcal{A}} \alpha \bar{t}_{a}+\hat{C} \leq \hat{F}_{\mathrm{TDP}}$ would be excluded from $\Xi$, minimizing/maximizing $v_{b}$ on $\Xi$ would be a feasibility-based strategy, in which no feasible link flow solution to P-TDP would be removed. Thus, when $\hat{F}_{\text {TDP }}$ is sufficiently high, no feasible solutions will be removed from TDP.

We will now proceed to describe how $\hat{C}$ can be estimated. Let $x^{0}$ be the no-toll solution to P-TDP (i.e. $\tau_{a}=0$ and $y_{a}=0, a \in \mathcal{A}$ ). If there exists a feasible solution to P-TDP with $F_{\mathrm{TDP}}(\bar{x}) \leq F_{\mathrm{TDP}}\left(x^{0}\right)$, then $\hat{C}$ must at least be equal to the cost of locating one toll on the link with lowest value on $\chi_{a}$, i.e. $\hat{C}=\min _{a \in \mathcal{A}} \chi_{a}$. If such a solution does not exist, $\hat{C}$, can always be underestimated by 0 . A tighter bound can be obtained by formulating and solving the following MILP

P-MILP-C: $\min \hat{C}$

$$
\begin{aligned}
& \text { subject to } \sum_{i \in \mathcal{I}} \Delta_{i}+\sum_{a \in \mathcal{A}}\left(\alpha \bar{t}_{a}+\chi_{a} y_{a}\right) \leq \hat{F}_{\mathrm{TDP}} \\
& \sum_{a \in \mathcal{A}} \chi_{a} y_{a} \leq \hat{C} \\
& \sum_{a \in \mathcal{A}}\left(\alpha \bar{t}_{a}+\sigma_{a}+\left(\alpha \theta_{a}-\tau_{a}\right) \hat{v}_{a}^{s}\right)+\sum_{i \in \mathcal{I}}\left(d_{i} \hat{q}_{i}^{s}+\delta_{i}\right) \leq 0, \quad s \in 1, \ldots, S \\
& v_{a}^{L} \leq v_{a} \leq v_{a}^{U}, \\
& a \in \mathcal{A} \\
& 0 \leq \tau_{a} \leq y_{a} \tau_{a}^{\max } \\
& a \in \mathcal{A} \\
& y_{a} \in\{0,1\} \text {, } \\
& a \in \mathcal{A} \\
& (v, q) \in \Omega \text {, } \\
& \text { and constraints (5), (6), (7), (8)and(9), }
\end{aligned}
$$

which essentially is P-MILP, but with the objective function of P-MILP introduced as constraint (11b), similar as in $\Xi$. If constraints (11a) and (11b) are disregarded, it is clear that an optimal solution to P-TDP must be feasible with respect to the remaining constraints, which essentially is the set $\Xi$ but with the VI constraints included. Constraint (11a) will restrict the objective function value of P-MILP by $\hat{F}_{\text {TDP }}$, but since 
P-MILP is a relaxation of P-TDP an optimal solution to P-MILP will always be feasible with respect to constraint (11a). Constraint (11b) will relate the lower bound estimate of the operator costs $\hat{C}$ to the actual cost. Minimizing $\hat{C}$ in P-MILP-C will give the lowest possible value on the total operator cost for which the objective function value to P-MILP is lower than $\hat{F}_{\text {TDP }}$.

The same branch and cut framework which was presented in Section 4 can be used for solving P-MILP-C. A lower bound, $\hat{C}^{\mathrm{LB}}$, of $\hat{C}$ can, however, be obtained at any time during the branch and cut process, and the problem does not need to be solved to optimality. If $\chi_{a}$ is equal for all links, i.e. $\chi_{a}=C$ for each link $a$, the optimal value $\hat{C}$ must be a multiple of $C$, and thus $\hat{C}^{\mathrm{LB}}$ can be rounded upwards to the closest multiple of $C$.

\subsection{Design strategies for choosing link flow break points}

Given $v_{a}^{L}$ and $v_{a}^{U}$, possibly obtained by domain reduction, there is still the matter of deciding the number of additional break points, and their values. Two different strategies are suggested and later evaluated.

For Strategy 1 we note that a good approximation in the vicinity of the optimal solution to P-TDP is desirable. Two known link flow solutions to P-TDP are the MSCP and the no-toll solutions. The MSCP solution gives SO link flows and demands, and if the operator costs are close to 0 we can expect the optimal solution, to P-TDP, to have a link flow solution close to the SO solution. If the operator costs are set sufficiently high, the optimal solution to P-TDP will be the no-toll solution. Thus, it is reasonable to assume that for an optimal solution to P-TDP, a majority of the links will have link flows between the SO and the no-toll link flows. The error introduced by the piecewise linear approximation can be viewed as a relaxation of the VI constraints. Thus it is a sound assumption that the link flow solution from an optimal solution to P-MILP will be closer to the SO link flow solution for approximations which introduce large errors in the VI constraints. Based on these observations, the following segmentation of the link flow space is suggested

$$
\begin{aligned}
J_{a, 0} & :=v_{a}^{L} \\
J_{a, 1} & :=\min \left\{v_{a}^{S O}, v_{a}^{S O}+\left(v_{a}^{0}-v_{a}^{S O}\right) \lambda\right\} \\
J_{a, 2} & :=\max \left\{v_{a}^{S O}, v_{a}^{S O}+\left(v_{a}^{0}-v_{a}^{S O}\right) \lambda\right\} \\
J_{a, 3} & :=v_{a}^{U}
\end{aligned}
$$

where $v^{S O}$ and $v_{a}^{0}$ are the link flow vectors corresponding to the SO link flows and no-toll link flows respectively, and $\lambda$ is a parameter chosen on the interval [0,1]. If $J_{a, 1} \leq v_{a}^{L}$ or $J_{a, 2} \geq v_{a}^{U}$ the breakpoint is removed, and if $v_{a}^{S O}=v_{a}^{0}=0, J_{a, 2}$ is removed and $J_{a, 1}=\left(v_{a}^{L}+v_{a}^{U}\right) / 2$. Note that $\lambda=1$ gives $J_{a, 2}:=v_{a}^{0}$, and $\lambda=0$ gives $J_{a, 2}=J_{a, 1}$, and the piecewise linear function can for the latter case be formulated with one single breakpoint, resulting in two linear segments for each link.

In Strategy 2, the link flow space, given by $v^{L}$ and $v^{U}$, will be equally divided into $\Lambda$ segments, not taking the SO and no-toll link flows into account. With a high number of segments, Strategy 2 is likely to give an approximation closer to the real non-linear functions, compared with Strategy 1, which is limited to three linear segments at most. 
Note that in both Strategy 1 and 2 the domain reduction for the link flow variables will play an important role. In Strategy 1 it will affect the error introduced by the piecewise linear segment between $J_{a, 0}$ and $J_{a, 1}$, and between $J_{a, 2}$ and $J_{a, 3}$. For Strategy 2 , the distance between the link flow break points is for link $a$ given by $\left(v_{a}^{U}-v_{a}^{L}\right) / \Lambda$, and will be reduced when $v_{a}^{L}$ is increased and when $v_{a}^{U}$ is decreased. Thus, the error introduced from the corresponding piecewise linear segments will also be reduced. The two strategies will be evaluated with respect to the quality of the solutions produced, as well as of the quality of their optimistic estimations of the optimal objective function value to $\mathrm{P}-\mathrm{TDP}$.

\section{$6 \quad$ Numerical results}

In this section numerical results are presented for the Sioux Falls network data from Yildirim (2001), with 87 links and 30 OD pairs (Figure 4). The connectors are assumed to not be tollable, which result in a total of 76 tollable links. The link specific operator cost is assumed to be equal for all links, i.e. $\chi_{a}=C$ for each link $a$, and given in the same unit as the toll levels. Three different operator costs are evaluated $(5,20,100)$. The relationship between travel time and traffic flow is given by

$$
t_{a}\left(v_{a}\right)=t_{a}^{0}+10^{-7} \zeta_{a} v_{a}^{4}
$$

where $t_{a}^{0}$ and $\zeta_{a}$ are link specific parameters. The value of time is assumed to be equal to one, i.e. $\alpha=1$. The inverse travel demand functions are on the form

$$
D_{i}^{-1}\left(q_{i}\right)=\beta \cdot\left(\psi_{i}-q_{i}\right)
$$

which is a linear function for each OD pair $i \in \mathcal{I}$, with $\psi_{i}$ being the maximum demand in OD pair $i$ and $\beta$ being a parameter equal to 2 for all OD pairs. Both link and demand data are given in Appendix B and can also be found in Yildirim (2001). The no-toll link flow solution can easily be obtained for the network by solving P-UE, and will always be feasible in P-TDP, with objective function value $F_{\mathrm{TDP}}^{0}=-6695$, link flow $v^{0}$ and demand $q^{0}$. The MSCP tolls give the system optimal demand and link flow distribution $\left(v^{\mathrm{SO}}, q^{\mathrm{SO}}\right)$, and assuming $\chi_{a}=0, a \in \mathcal{A}, F_{\mathrm{TDP}}^{\mathrm{MSCP}}=-9416.74$, with tolls on 47 links. Yildirim (2001) finds the SO solution with only 24 tolls located, but in this solution it is allowed to toll connectors, thus this solution will not be directly comparable with the results presented here. Demand and link flow solutions for the UE and SO cases are given in Appendix B.

The points in link flow space, used for linearizing the total travel time functions, are link specific and given as functions of the link capacity, $T_{a}^{k}=c_{a}(k-1) / 10, k \in 1, \ldots, 46$. Setting $k=46$ gives $T_{a}^{k}=4.5 c_{a}$, i.e. it is assumed that the flow will not exceed 4.5 times the capacity in any solution. Also, $T_{a}^{47}=v_{a}^{\mathrm{SO}}, T_{a}^{48}=v_{a}^{0}$ are included. For $-D_{i}^{-1}\left(q_{i}\right) q_{i}$ and $-\int_{0}^{q_{i}} D_{i}^{-1}(w) d w$, the points of linearization, $Q_{i}^{m}$, are given as function of the maximum demand $\psi_{i}, Q_{i}^{m}=\psi_{i}(m-1) / 20, m \in 1, \ldots, 20$. Note that $m=20$ gives $Q_{i}^{m}$ equal to the maximum demand $\psi_{i}$. Similar as for the total travel time function, $Q_{i}^{21}=q_{i}^{\mathrm{SO}}, Q_{i}^{42}=q_{i}^{0}$ are also included. To include the SO link flows and demands will ensure that the optimal objective function value to P-MILP will be equal to or higher than the social surplus associated with SO link flows and demands (see discussion in 


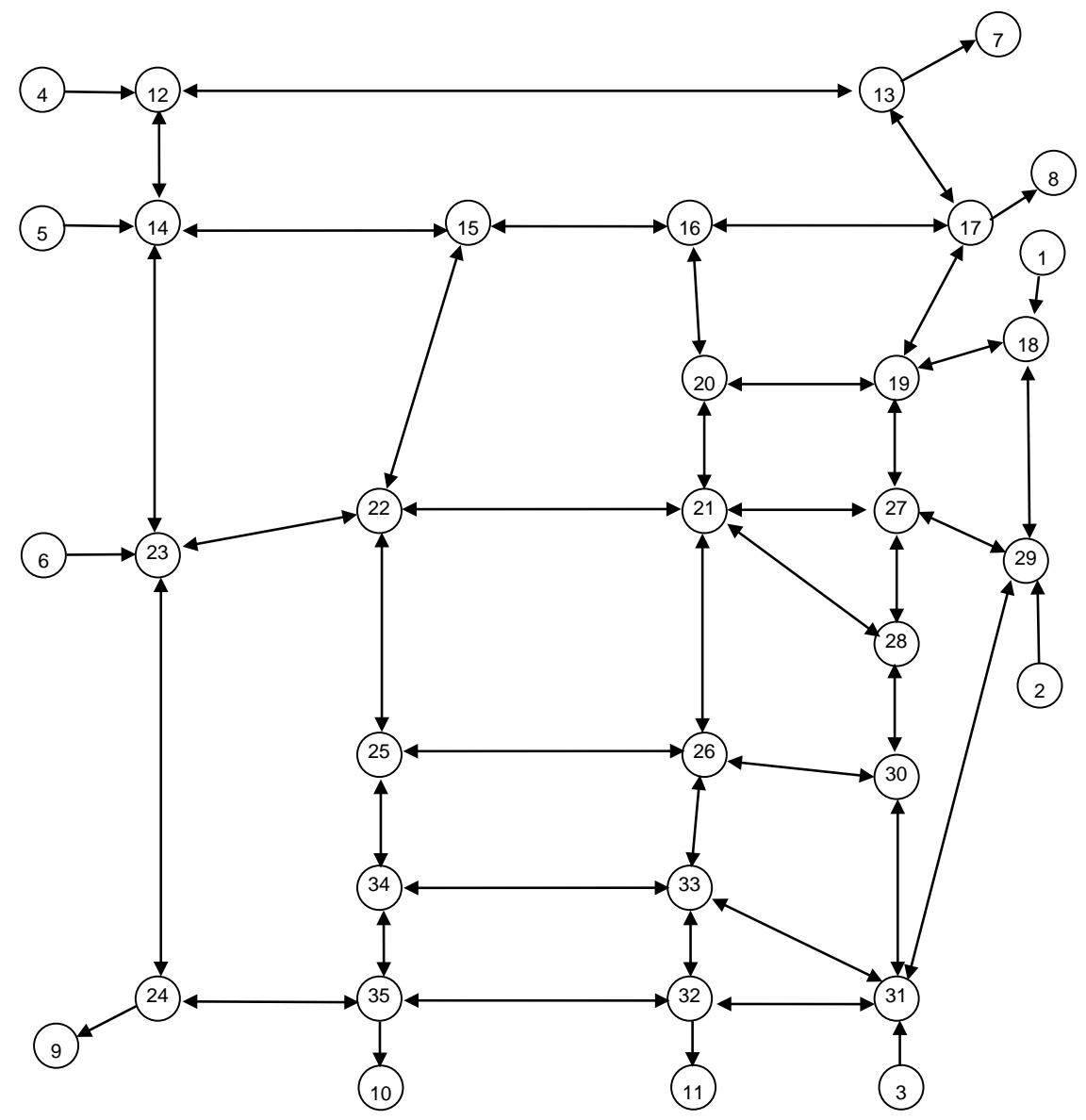

Figure 4: The Sioux Falls network.

Section 3.4). For Strategy 1, computations are presented for three different values on $\lambda$; $0,0.1$, and 0.4. $\lambda=0$ gives an approximation scheme with two link flow segments for each link, and $\lambda>0$ gives approximation schemes with three link flow segments for each link. For Strategy 2, three different segmentations of the link flow space are evaluated; the first one with $\Lambda=3$, the second one with $\Lambda=6$, and the third one with $\Lambda=12$.

For solving P-MILP and P-MILP-C, the CPLEX solver (version 12.2) is used, in which the node subproblems are solved with SP-CCA, as described in Section 4. The integration of SP-CCA with CPLEX is done with IBM ILOG CPLEX Concert technology in Java and one single core of an Intel P8600 2.40GHz CPU is used for the computations. Different settings can be used to control the behaviour of CPLEX and SP-CCA. For finding feasible solutions, the parameter $\epsilon$ is set to 1500 in SP-CCA, and in CPLEX the priority is set to find feasible solutions rather than proving optimality. To improve the bound of P-TDP, $\epsilon$ is set to zero and in CPLEX the priority is set to move the best bound.

P-MILP will for each combination of approximation scheme and operator cost, be solved with three different link flow bounds. The link flow bounds are computed by 
minimizing/maximizing $v_{b}$ over $\Xi$ as is described in Section 5.1.

1. $v^{U}$ and $v^{L}$ computed with $\hat{F}=F_{\text {TDP }}^{0}$ and $\hat{C}=0$.

2. $v^{U}$ and $v^{L}$ computed with $\hat{F}$ equal to the best found solution so far, and $\hat{C}=C$.

3. $v^{U}$ and $v^{L}$ computed with $\hat{F}$ equal to the best found solution so far, and the highest value of $\hat{C}$ obtained by solving P-MILP-C, with each approximation scheme, for $1800 \mathrm{CPU}$-seconds and rounding $\hat{C}^{L B}$ upward to the closest multiple of $C$.

P-TDP is, for each approximation scheme, solved with priority to find good feasible solutions and with a time limit set to $1800 \mathrm{CPU}$-seconds. The resulting solution is denoted $\tilde{x}$ with objective function value $F_{\mathrm{MILP}}(\tilde{x})$ (Table 1 ). If optimality is not proven within this time limit, $F_{\mathrm{MILP}}(\tilde{x})$ cannot be used as lower bound estimation on the objective function value to P-TDP. The best found lower bound to the optimal objective function value to P-MILP is however a valid lower bound to the optimal objective function value to P-TDP. To find a strong lower bound P-MILP is therefore also, for each combination of approximation scheme and $C$, solved for another $1800 \mathrm{CPU}$-seconds with priority to increase the lower bound of the optimal objective function value to P-MILP (presented in Table 2), rather than finding feasible solutions.

Table 1: P-MILP objective function value $\left(F_{\mathrm{MILP}}(\tilde{x})\right)$ and the number of located tolls (in parenthesis)

\begin{tabular}{ccccccccc}
\hline \hline & Input & \multicolumn{3}{c}{ Strategy 1} \\
$C$ & $\hat{F}$ & $\hat{C}$ & $\lambda=0$ & $\lambda=0.1$ & $\lambda=0.4$ & $\Lambda=3$ & $\Lambda=6$ & $\Lambda=12$ \\
\hline 5 & -6695 & 0 & $-9362(10)$ & $-9355(12)$ & $-9358(11)$ & $-9382(7)$ & $-9367(10)$ & $-9362(11)$ \\
5 & -9283 & 5 & $-9358(12)$ & $-9355(12)$ & $-9357(11)$ & $-9360(11)$ & $-9359(11)$ & $-9346(13)$ \\
5 & -9297 & 30 & $-9357(12)$ & $-9354(12)$ & $-9357(11)$ & $-9360(11)$ & $-9361(11)$ & $-9346(14)$ \\
\hline 20 & -6695 & 0 & $-9248(7)$ & $-9214(9)$ & $-9193(11)$ & $-9277(7)$ & $-9216(10)$ & $-9173(12)$ \\
20 & -9106 & 20 & $-9232(7)$ & $-9183(11)$ & $-9186(11)$ & $-9223(9)$ & $-9175(11)$ & $-9171(12)$ \\
20 & -9106 & 80 & $-9217(7)$ & $-9180(10)$ & $-9183(11)$ & $-9214(8)$ & $-9175(12)$ & $-9148(12)$ \\
\hline 100 & -6695 & 0 & $-8892(4)$ & $-8683(6)$ & $-8582(7)$ & $-8784(6)$ & $-8671(7)$ & no solution $^{1}$ \\
100 & -8501 & 100 & $-8800(5)$ & $-8658(7)$ & $-8565(7)$ & $-8755(6)$ & $-8659(7)$ & no solution $^{1}$ \\
\hline \hline
\end{tabular}

${ }^{1}$ No feasible solution obtained within time limit

There are several methods which, given a fixed set of toll locations, can be used to search for local optimal toll levels, for example Verhoef (2002), Lawphongpanich and Hearn (2004) and Ekström et al. (2009). Local optimal search methods are for nonconvex problems sensitive to the initial solution, and the toll levels from P-MILP can therefore be used as an initial solution, when polishing the toll levels to further improve the P-TDP solution.

The toll location and toll level solution from P-MILP, $(\tilde{\tau}, \tilde{y})$, has been applied to P-TDP, with objective function value denoted as $F_{\mathrm{TDP}}(\tilde{\tau}, \tilde{y})$ (given in Table 3 ), and then further polished by the local optimal search method from Ekström et al. (2009), resulting in toll levels $\tilde{\tau}^{\mathrm{LS}}$ with objective function value $F_{\mathrm{TDP}}\left(\tilde{\tau}^{\mathrm{LS}}, \tilde{y}\right)$ (given in Table 4 ). The toll level solution corresponding to the best found solution, after polishing by local optimal search, can be found in Appendix B (for each value on $C$ ). For comparison, the smoothing heuristic presented in Ekström et al. (2009) is also used for each value on $C$. 
Table 2: Lower bound of the optimal objective function value to P-TDP, presented for each combination of approximation scheme and operator cost

\begin{tabular}{ccccccccc}
\hline \hline & Input & \multicolumn{3}{c}{ Strategy 1} & \multicolumn{3}{c}{ Strategy 2} \\
$C$ & $\hat{F}$ & $\hat{C}$ & $\lambda=0$ & $\lambda=0.1$ & $\lambda=0.4$ & $\Lambda=3$ & $\Lambda=6$ & $\Lambda=12$ \\
\hline 5 & -6695 & 0 & -9391 & -9393 & -9387 & -9387 & -9383 & -9379 \\
5 & -9283 & 5 & -9379 & -9380 & -9380 & -9379 & -9380 & -9381 \\
5 & -9297 & 30 & -9379 & -9379 & -9379 & -9379 & -9378 & -9382 \\
\hline 20 & -6695 & 0 & -9339 & -9346 & -9327 & -9307 & -9298 & -9293 \\
20 & -9106 & 20 & -9289 & -9300 & -9295 & -9294 & -9287 & -9293 \\
20 & -9106 & 80 & -9288 & -9293 & -9291 & -9292 & -9287 & -9282 \\
\hline 100 & -6695 & 0 & -9218 & -9223 & -9212 & -9005 & -8948 & -9001 \\
100 & -8501 & 100 & -9095 & -9110 & -9083 & -9004 & -8932 & -8968 \\
\hline \hline
\end{tabular}

The relative gap between the best found solution to P-TDP $(U B)$ and the highest lower bound estimation $(L B)$ of its objective function value is presented in Table 5 , where the relative gap is computed as

$$
g a p=\frac{L B-U B}{L B} .
$$

As a measure of the approximation quality the mean relative error of the travel time approximations (presented in Table 6) are used, and computed as

$$
e=\frac{1}{n} \sum_{a \in \mathcal{A}} \frac{-\theta_{a}-t_{a}\left(\tilde{v}_{a}\right)}{t_{a}\left(\tilde{v}_{a}\right)}
$$

where $n$ is the number of links with non-constant travel time functions, and $\tilde{v}_{a}$ is the link flow solution to P-MILP.

Table 3: Objective function value to P-TDP when applying the toll level solution from P-MILP $\left(F_{\mathrm{TDP}}(\tilde{\tau}, \tilde{y})\right)$, best solution for each $C$ is marked in bold

\begin{tabular}{ccccccccc}
\hline \hline & Input & \multicolumn{3}{c}{ Strategy 1} & \multicolumn{3}{c}{ Strategy 2} \\
$C$ & $\hat{F}$ & $\hat{C}$ & $\lambda=0$ & $\lambda=0.1$ & $\lambda=0.4$ & $\Lambda=3$ & $\Lambda=6$ & $\Lambda=12$ \\
\hline 5 & -6695 & 0 & -9099 & -9254 & -9213 & -9138 & -9072 & -9215 \\
5 & -9283 & 5 & -9238 & -9241 & -9225 & -9056 & -9227 & -9201 \\
5 & -9283 & 30 & -9178 & -9240 & -9259 & -9196 & -9207 & $\mathbf{- 9 3 0 0}$ \\
\hline 20 & -6695 & 0 & -8888 & -8924 & $\mathbf{- 9 0 5 8}$ & -9029 & -8922 & -9008 \\
20 & -9106 & 20 & -9046 & -9053 & -9044 & -8988 & -8892 & -9055 \\
20 & -9106 & 80 & -9018 & -8760 & -9033 & -8996 & -8998 & -9044 \\
\hline 100 & -6695 & 0 & -7910 & -8425 & -8466 & -8311 & $-\mathbf{8 4 8 7}$ & no solution $^{1}$ \\
100 & -8501 & 100 & -8078 & $\mathbf{- 8 4 8 7}$ & -8461 & -8298 & -8481 & no solution \\
\hline \hline
\end{tabular}

${ }^{1}$ No solution to P-MILP obtained within time limit

Optimality is not proven for any of the solutions to P-MILP, and there is no guarantee that the obtained solution is the optimal one. For $C=5$ with $\Lambda=6$ it is clear from 
Table 4: Objective function value to P-TDP, after polishing by local search heuristic $\left(F_{\mathrm{TDP}}\left(\tilde{\tau}^{\mathrm{LS}}, \tilde{y}\right)\right)$, best solution for each $C$ is marked in bold

\begin{tabular}{ccccccccc}
\hline \hline & Input & \multicolumn{3}{c}{ Strategy 1} & \multicolumn{3}{c}{ Strategy 2} \\
$C$ & $\hat{F}$ & $\hat{C}$ & $\lambda=0$ & $\lambda=0.1$ & $\lambda=0.4$ & $\Lambda=3$ & $\Lambda=6$ & $\Lambda=12$ \\
\hline 5 & -6695 & 0 & -9233 & -9283 & -9271 & -9166 & -9177 & -9267 \\
5 & -9283 & 5 & -9266 & -9297 & -9271 & -9228 & -9270 & -9273 \\
5 & -9283 & 30 & -9270 & -9287 & -9283 & -9241 & -9270 & $\mathbf{- 9 3 2 3}$ \\
\hline 20 & -6695 & 0 & -8958 & -9084 & $\mathbf{- 9 1 0 6}$ & -9061 & -9029 & -9056 \\
20 & -9106 & 20 & -9061 & $\mathbf{- 9 1 0 6}$ & $\mathbf{- 9 1 0 6}$ & -9069 & -8991 & -9073 \\
20 & -9106 & 80 & -9061 & -9071 & $\mathbf{- 9 0 7 9}$ & -9052 & $\mathbf{- 9 0 5 6}$ & -9082 \\
\hline 100 & -6695 & 0 & -7926 & -8463 & $\mathbf{- 8 5 0 1}$ & -8374 & $\mathbf{- 8 5 0 1}$ & no solution $^{1}$ \\
100 & -8501 & 100 & -8126 & $\mathbf{- 8 5 0 1}$ & $\mathbf{- 8 5 0 1}$ & -8314 & $\mathbf{- 8 5 0 1}$ & no solution \\
\hline \hline
\end{tabular}

${ }^{1}$ No feasible solution from P-MILP available to polish

Table 5: Best found upper bound $(U B)$ estimation, best found lower bound estimation $(L B)$ of the optimal objective function value to P-TDP for each $C$, with corresponding relative gap (gap)

\begin{tabular}{cccc}
\hline \hline$C$ & $U B$ & $L B$ & gap $(\%)$ \\
\hline 5 & -9323 & -9378 & 0.59 \\
20 & -9106 & -9287 & 1.9 \\
100 & -8501 & -8932 & 4.8 \\
\hline \hline
\end{tabular}

Table 1, comparing the results for $\hat{C}=5(1)$ and $\hat{C}=30(2)$, that the solution $\tilde{x}^{(1)}$ with $F_{\text {MILP }}\left(\tilde{x}^{(1)}\right)=-9359$ cannot be optimal since another solution, $\tilde{x}^{(2)}$, is found with $F_{\text {MILP }}\left(\tilde{x}^{(2)}\right)=-9361$. Note that using $\hat{C}$ instead of $C$ when computing $v^{L}$ and $v^{U}$, used in the approximation schemes, will reduce the feasible area of P-MILP, thus we should have $F_{\mathrm{MLP}}\left(\tilde{x}^{(1)}\right) \leq F_{\mathrm{MILP}}\left(\tilde{x}^{(2)}\right)$ if the two solutions were optimal. For all other cases the objective function value to P-MILP is increased when the link flow bounds are updated, although there is no guarantee that the optimal solution is obtained. The relative gap between the best found feasible solution and the lower bound estimation is presented in Table 5, and comparing the relative gap for each value on $C$, it is clear that when $C$ is reduced so is the relative gap. Since the SO link flow and demand solution is used in P-MILP for linearizing the objective function in P-TDP, P-MILP and P-TDP will have equal optimal objective function values at $C=0$. Thus the relative gap is expected to tend towards zero as $C$ tends towards zero.

For $C=20$ the best found solution, when applying $(\tilde{\tau}, \tilde{y})$ to $\mathrm{P}$-TDP is with the initial approximation scheme (Strategy 1 with $\lambda=0.4$ ). Strategy 1 , with $\lambda>0$, in general result in small mean relative errors in the approximation of travel times, but do not necessarily produce toll level solutions which result in the best solutions to P-TDP. For $C=5$, Strategy 2 with $\Lambda=12$ outperforms all other results. It is, however, difficult to make any definitive statement about which strategy is the better one based on the results presented here. The solution which gives the lowest objective function values to P-TDP, for each $C$, also leads to the best solutions after the local optimal search is 
Table 6: Mean relative error in the approximation of travel time functions

\begin{tabular}{ccccccccc}
\hline \hline & Input & \multicolumn{3}{c}{ Strategy 1} & \multicolumn{3}{c}{ Strategy 2} \\
$C$ & $\hat{F}$ & $\hat{C}$ & $\lambda=0$ & $\lambda=0.1$ & $\lambda=0.4$ & $\Lambda=3$ & $\Lambda=6$ & $\Lambda=12$ \\
\hline 5 & -6695 & 0 & 0.047 & 0.0039 & 0.0070 & 0.069 & 0.015 & 0.0035 \\
5 & -9283 & 5 & 0.0070 & 0.0019 & 0.0034 & 0.014 & 0.0036 & 0.00084 \\
5 & -9283 & 30 & 0.0062 & 0.0026 & 0.0028 & 0.011 & 0.0034 & 0.00070 \\
\hline 20 & -6695 & 0 & 0.062 & 0.027 & 0.0079 & 0.070 & 0.016 & 0.0039 \\
20 & -9106 & 20 & 0.029 & 0.0074 & 0.0053 & 0.028 & 0.0073 & 0.0019 \\
20 & -9106 & 80 & 0.023 & 0.0081 & 0.0067 & 0.024 & 0.0061 & 0.0014 \\
\hline 100 & -6695 & 0 & 0.16 & 0.046 & 0.039 & 0.069 & 0.018 & - \\
100 & -8501 & 100 & 0.097 & 0.032 & 0.014 & 0.051 & 0.013 & - \\
\hline \hline
\end{tabular}

applied. Overall, the benefit from applying local optimal search is small, which suggest that the solutions to P-MILP are not far from the closest local optima.

It is interesting to compare the best found solution for each value on $C$, with the worst solution found with Strategy 1 (separated for $\lambda=0$ and $\lambda>0$ ), and for Strategy 2. The deviation from the best found solution is between $0.97 \%$ and $6.76 \%$ for Strategy 1 with $\lambda=0$, between $0.38 \%$ and $0.68 \%$ for Strategy 1 with $\lambda>0$, and between $1.26 \%$ and $2.20 \%$ for Strategy 2. Thus Strategy 1 with $\lambda>0$ seems to be more reliable in producing good solutions.

For $C=5$ the smoothening heuristic from Ekström et al. (2009) gives $F\left(\tau^{\mathrm{S}}, y^{\mathrm{S}}\right)=$ -9315 with 20 located tolls, for $C=20$ the resulting objective function value is $F\left(\tau^{\mathrm{S}}, y^{\mathrm{S}}\right)=-9066$ with 17 located tolls, and for $C=100$ the resulting objective function value is $F\left(\tau^{\mathrm{S}}, y^{\mathrm{S}}\right)=-8304$ with 8 located tolls. Thus better solutions are obtained with the polished solutions from P-MILP, for all values on $C$, compared with the smoothening heuristic. Since the polishing is only performed on the toll levels, it seems that solutions to P-MILP achieve better toll location solutions, at least for the Sioux Falls network used in this paper.

Strategy 1 with $\lambda=0$ and Strategy 2 with $\Lambda=3$ stand out as the approximation schemes which in general perform worse, especially for $C=100$. These schemes also correspond with a high mean relative error in the approximation of the travel times (Table 6). While Strategy 2 with $\Lambda=3$ and Strategy 1 with $\lambda>0$ have an equal number of linear segments the choice of break points in Strategy 1 clearly improve the approximation quality. Considering the lower bound estimation of the optimal objective function value to P-TDP in Table 2, Strategy 2 in general performs best. The effect of the link flow bound update is, however, more noticeable for Strategy 1, but the effect of updating $\hat{C}$, before applying domain reduction, is small for all cases.

To analyze the differences between the different solutions obtained by the two strategies, and their different parameter values, Figure 5 shows the number of times each toll location appears in a solution. From Figure 5 it is clear that there are differences in the toll locations, between the solutions. There are only two links (link 45 and 71), which appear in all of the solutions. Another comparison can be made by considering the MSCP toll level solution. Comparing the best obtained solutions, given in Table 9, with the toll locations with highest toll levels in the MSCP solution gives the following 
results. For $C=5$, out of 14 located tolls, 3 are not among the 14 tolls with highest MSCP toll level, for $C=20$ and $C=100$, there are also 3 toll locations which are not among the 11 and 7 tolls, respectively, with highest MSCP toll level. Thus, as fewer tolls are located, a larger proportion of these tolls are not among the toll locations which have highest MSCP toll level.

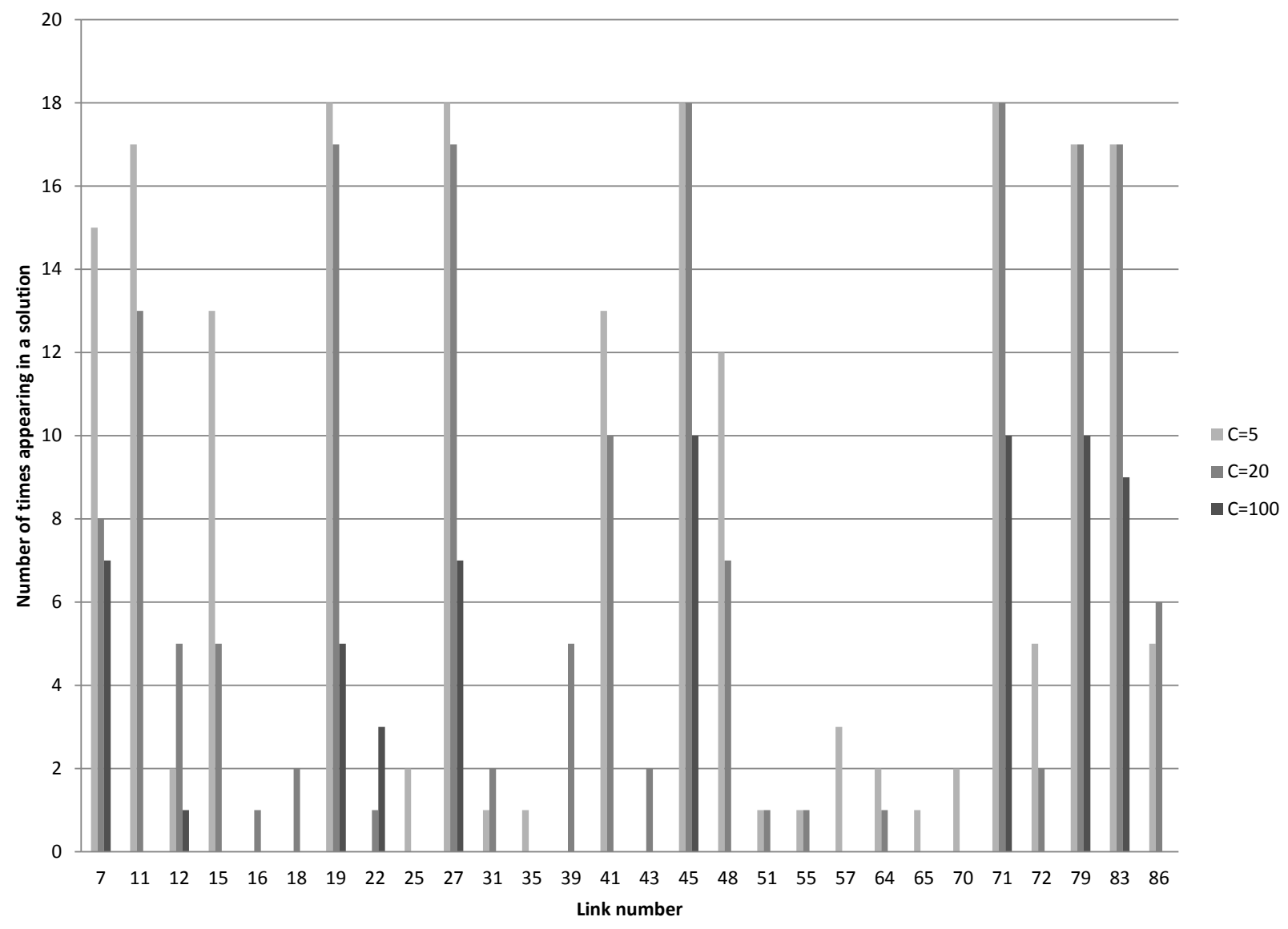

Figure 5: Number of times each link toll is appearing in a solution, separated for each value on $C$.

Finally, considering the resulting toll locations and levels, it is interesting but not surprising, to see that with $C=100$, the tolls are located so that every possible route in every OD pair is tolled once. Thus, the benefit from the located tolls is mainly related to reduced demand. As $C$ is increased, additional tolls are located in order to achieve benefits from optimal routing.

\section{Discussion}

In this paper a piecewise linear approximation of the toll design problem has been presented. The piecewise linear approximation requires a number of binary variables to 
be introduced, and the resulting problem is a MILP. The MILP formulation relies on a large number of VI constraints, which makes it difficult to directly use commercially available solvers. In Ekström et al. (2012) the MILP (formulated for a fixed demand network) is solved by a CCA, which requires one MILP to be solved to optimality in each iteration. Already for the medium sized network used in this paper this approach cannot be used in practice, due to the computational burden of solving one MILP to optimality in each iteration. In this paper we have instead presented a branch and cut framework, in which each subproblem is solved with a CCA (SP-CCA). The main benefits are that (1) any integer solution returned during the branch and cut process is a feasible solution to P-MILP, (2) any lower bound estimation of the optimal objective function value to P-MILP, obtained during the branch and cut process, is a valid lower bound of the optimal objective function value to P-TDP, and (3) SP-CCA can be integrated with commercially available solvers.

To improve the MILP approximation, domain reduction has been applied, to reduce the link flow space, and thus the error introduced by piecewise linear approximations of the non-convex functions. Two different strategies for choosing link flow break points have been evaluated. For Strategy 1, when the break points are chosen based on the SO and no-toll link flow solutions, domain reduction has a larger effect on the lower bound estimation of the optimal objective function value to P-TDP, while domain reduction combined with Strategy 2, when the break points are evenly distributed, has a less effect. For both strategies, the error in travel time approximations is clearly reduced when domain reduction based on the best found feasible solution to P-TDP is applied.

The approximation error can be viewed as a relaxation of the VI constraints (i.e. a relaxation of the UE condition), with the effect that a link flow solution which would require a certain number of located tolls to be an equilibrium solution, can be achieved with a fewer number of tolls located. In general, an approximation scheme with a small travel time approximation error has more tolls located compared to an approximation scheme with a high error. On the other hand, increasing the number of linear segments (i.e. increasing the number of binary variables) in Strategy 2 to reduce the error will increase the time needed to solve the optimization problem. By applying domain reduction it is possible to use only three link flow segments with Strategy 1, with roughly the same travel time approximation error as with Strategy 2 and six link flow segments (i.e. more than twice as many binary variables).

In terms of lower bound estimations of the optimal objective function value to $\mathrm{P}$ TDP, obtained by solving P-MILP, the choice of strategy for selecting the link flow break points does not have a large impact on the quality of these estimations. Both Strategy 1 and Strategy 2 perform well, but it seems that Strategy 2 has some advantage when the number of located tolls are reduced (as $C$ is increased). This is an expected result as the link flow solution will be closer to the SO solution when $C$ is low. Strategy 1 with $\lambda=0$ (i.e. only one break point), will benefit the most from domain reduction, and will for $C=5$ and $C=20$ return lower bounds equal to or better than what is obtained with $\lambda>0$. Using only one break point will also reduce the complexity of P-MILP, and therefore Strategy 1 with $\lambda=0$ could be an interesting choice when applying the approach to larger networks, given that domain reduction is applied. To update the underestimation of the operator costs $(\hat{C})$, before applying domain reduction, has however shown to only give minor positive effects on the lower bound estimations and 
solution qualities, and for some cases even negative effects. For the quality of the toll locations and toll levels, when being evaluated in P-TDP, the results are not conclusive on which choice of strategy and parameter setting that will generate good solutions. It is, however, clear that Strategy 1 with $\lambda=0$ overall perform worse compared with the other choices of parameter settings. One problem here could be that optimality is not proven for the solutions to P-MILP, and it is therefore possible that there exist better solutions to P-MILP which have not been found.

To solve P-MILP to optimality for larger networks can at the present time not be done within a reasonable time. To develop heuristic approaches for finding good feasible solutions to P-MILP, which could make use of the special structure of the problem, could improve the solution quality especially for larger networks. Remember that any lower bound to P-MILP, obtained during the branch and cut process (see Section 3.4), will be equal to or better then what can be computed based on SO link flows and demands. For practical applications a lower bound could then be obtained from applying the branch and cut framework to solve P-MILP (with the quality of the lower bound depending on the available solution time), and an upper bound could be obtained by applying a tailored heuristic to find good feasible solutions to P-MILP.

In this paper piecewise linear functions have been used to approximate the non-linear travel time functions. There is no guarantee that the original travel time function gives the best description of the relationship between travel time and traffic flow, and it is possible to instead directly use piecewise linear functions to describe this relationship. While this would eliminate the error introduced by the approximation of the travel time functions, there is still the need for approximating total travel time functions, link toll revenue functions and non-linear functions related to travel demand. The use of piecewise linear travel time functions is straightforward and the same framework as presented in this paper, for solving P-MILP, can be used.

It is possible to extend the TDP formulation to multiple user classes and/or nonlinear pricing. The introduction of multiple user classes would make it possible to take differentiated values of time, among the road users, into account, and non-linear pricing opens for applications to a wider range of congestion pricing schemes.

\section{Acknowledgements}

The presentation of this paper benefited from the comments of three anonymous reviewers. The project is also supported by the Internal Research Grant of The Hong Kong Polytechnic University (G-UA04).

\section{References}

Al-Khayyal, F.A. and Falk, J.E., 1983. Jointly constrained biconvex programming. Mathematics of Operations Research, 8 (2), 273-286.

Beckmann, M., McGuire, C., and Winsten, C.B., 1956. Studies in the Economics of Transportation. New Haven: Yale University Press.

Bureau of Public Roads, 1964. Traffic Assignment Manual. . 
Caprara, A. and Locatelli, M., 2010. Global optimization problems and domain reduction strategies. Mathematical Programming, 125, 123-137.

Dafermos, S., 1980. The general multimodal network equilibrium problem with elastic demand. Networks, 12 (1), 57-72.

Dantzig, G.B., 1963. Linear Programming and Extensions. Princeton, New Jersey: Princeton University Press.

Ekström, J., Engelson, L., and Rydergren, C., 2009. Heuristic algorithms for a secondbest congestion pricing problem. NETNOMICS: Economic Research and Electronic Networking, 10 (1), 85-102.

Ekström, J., Sumalee, A., and Lo, H.K., 2012. Optimizing toll locations and levels using a mixed integer linear approximation approach. Transportation Research Part B, 46 (7), 834-854.

Farvaresh, H. and Sepehri, M.M., 2011. A single-level mixed integer linear formulation for a bi-level discrete network design problem. Transportation Research Part E, 47 (5), $623-640$.

Floudas, C., 2000. Deterministic global optimization: theory, methods, and applications. Nonconvex optimization and its applications Kluwer Academic Publishers.

Gurobi Optimization, 2012. Gurobi Optimizer Reference Manual. .

IBM, 2010. IBM ILOG CPLEX V12.1 User's Manual for CPLEX. .

Koh, A., Shepherd, S., and Watling, D., 2012. Competition between two cities using cordon tolls: an exploration of response surfaces and possibilities for collusion. Transportmetrica, Available online.

Lawphongpanich, S. and Hearn, D.W., 2004. An MPEC approach to second-best toll pricing. Mathematical Programming, 101 (1), 33-55.

Luathep, P., et al., 2011. Global optimization method for mixed transportation network design problem: A mixed-integer linear programming approach. Transportation Research Part B, 45 (5), 808 - 827.

Marcotte, P., 1983. Network Optimization with Continuous Control Parameters. Transportation Science, 17 (2), 181 - 197.

Padberg, M., 2000. Approximating separable nonlinear functions via mixed zero-one programs. Operations Research Letters, 27 (1), $1-5$.

Sheffi, Y., 1985. Urban Transportation Networks: Equilibrium Analysis with Mathematical Programming Methods. New Jersey: Prentice-Hall.

Shepherd, S. and Sumalee, A., 2004. A genetic algorithm based approach to optimal toll level and location problems. Networks and Spatial Economics, 4 (2), 161-179. 
Sumalee, A., 2007. Multi-concentric optimal charging cordon design. Transportmetrica, $3(1), 41-71$.

Tawarmalani, M. and Sahinidis, N., 2002. Convexification and global optimization in continuous and mixed-integer nonlinear programming: theory, algorithms, software, and applications. Nonconvex optimization and its applications Kluwer Academic Publishers.

Tsekeris, T. and Voß, S., 2009. Design and evaluation of road pricing: state-of-theart and methodological advances. NETNOMICS: Economic Research and Electronic Networking, 10, 5-52.

Verhoef, E.T., 2002. Second-best congestion pricing in general networks. Heuristic algorithms for finding second-best optimal toll levels and toll points. Transportation Research Part B, 36 (8), 707-729.

Wang, D.Z. and Lo, H.K., 2010. Global Optimum of the Continuous Network Design Problem with Equilibrium Flows. Transportation Research Part B, 44 (4), $482-492$.

Wicaksono, D.S. and Karimi, I.A., 2008. Piecewise MILP under- and overestimators for global optimization of bilinear programs. AICHe Journal, 54 (4), 991-1008.

Wolsey, L.A. and Nemhauser, G.L., 1999. Integer and Combinatorial Optimization. 1 Wiley-Interscience.

Yang, H. and Zhang, X., 2003. Optimal Toll Design in Second-Best Link-Based Congestion Pricing. Transportation Research Records, 1857, 85-92.

Yildirim, B. and Hearn, D.W., 2005. A first best toll pricing framework for variable demand traffic assignment problems. Transportation Research Part B, 39, 659-678.

Yildirim, M.B., 2001. Congestion Toll Pricing Models and Methods for Variable Demand Networks. Thesis (PhD). University of Florida, Gainsville.

Zerbe, R.O.J. and Dively, D.D., 1994. Benefit-cost analysis in theory and practice. New York: HarperCollins.

Zhang, X. and van Wee, B., 2012. Enhancing transportation network capacity by congestion pricing with simultaneous toll location and toll level optimization. Engineering Optimization, 44 (4), 477-488.

Zhang, X., et al., 2011. Competitive, cooperative and Stackelberg congestion pricing for multiple regions in transportation networks. Transportmetrica, 7 (4), 297-320. 


\section{A Formulated optimization problems}

Table 7: Formulated optimization problems with main inputs and outputs

\begin{tabular}{|c|c|c|c|c|}
\hline Abbrivation & Description & $\begin{array}{l}\text { Variables and constraints } \\
\text { for picewise-linearization } \\
\text { of non-linear constraints } \\
\text { included }\end{array}$ & $\begin{array}{l}\text { Main } \\
\text { input }\end{array}$ & $\begin{array}{l}\text { Main } \\
\text { output }\end{array}$ \\
\hline \multicolumn{5}{|c|}{ Main optimization problem } \\
\hline P-UE & $\begin{array}{l}\text { The user equilibrium } \\
\text { problem }\end{array}$ & no & $\tau$ & $q, v$ \\
\hline $\mathrm{P}-\mathrm{SO}$ & $\begin{array}{l}\text { The system optimal } \\
\text { problem }\end{array}$ & no & & $q, v$ \\
\hline P-TDP & $\begin{array}{l}\text { The toll design } \\
\text { problem }\end{array}$ & no & $\chi, \tau^{\max }$ & $\tau, q, v, y$ \\
\hline P-MILP & $\begin{array}{l}\text { Relaxation of P-TDP } \\
\text { obtained by replacing } \\
\text { the non-linear function } \\
\text { in P-TDP by piecewise } \\
\text { linear ones }\end{array}$ & yes & $\chi, \tau^{\max }$ & $\tau, q, v, y$ \\
\hline \multicolumn{5}{|c|}{ Subproblems in the branch and cut algorithm } \\
\hline P-SP & $\begin{array}{l}\text { Node subproblem } \\
\text { of P-MILP in node } n\end{array}$ & yes & $\begin{array}{c}\Phi_{n}, \tau^{\max } \\
v^{L}, v^{U}\end{array}$ & $\tau, q, v, y$ \\
\hline $\mathrm{P}-\mathrm{SP}^{\prime}$ & $\begin{array}{l}\text { P-SP with reduced num- } \\
\text { ber of VI-constraints, } \\
\text { used in SP-CCA }\end{array}$ & yes & $\begin{array}{c}\Phi_{n}, R_{n} \\
\tau^{\max }, v^{L}, v^{U}\end{array}$ & $\begin{array}{c}\delta^{*}, \sigma^{*}, \bar{t}^{*} \\
\theta^{*}, d^{*}\end{array}$ \\
\hline P-LP & $\begin{array}{l}\text { Subproblem for finding } \\
\text { violated VI-constraints } \\
\text { in SP-CCA }\end{array}$ & yes & $\delta^{*}, \sigma^{*}, \bar{t}^{*}, \theta^{*}, d^{*}$ & $\tilde{q}, \tilde{v}$ \\
\hline \multicolumn{5}{|c|}{ Subproblems formulated for preprocessing } \\
\hline P-MILP-R & $\begin{array}{l}\text { P-MILP with the } \\
\text { VI-constrints removed }\end{array}$ & yes & $\begin{array}{l}\text { Used in Proposi } \\
\text { the domain red }\end{array}$ & $\begin{array}{l}1 \text { for developing } \\
\text { scheme }\end{array}$ \\
\hline P-MILP-C & $\begin{array}{l}\text { Bounding of the total } \\
\text { operator costs }\end{array}$ & yes & $\hat{F}_{\mathrm{TDP}}$ & $\hat{C}$ \\
\hline
\end{tabular}




\section{B Sioux Falls network data}

Table 8: Demand data and solution for each OD pair in the Sioux Falls network

\begin{tabular}{|c|c|c|c|c|}
\hline Origin & Destination & $\psi$ & $q^{0}$ & $q^{\mathrm{SO}}$ \\
\hline 1 & 7 & 40 & 15.68 & 10.87 \\
\hline 1 & 8 & 1 & 0.00 & 0.00 \\
\hline 1 & 9 & 48 & 22.16 & 16.50 \\
\hline 1 & 10 & 12 & 0.00 & 0.00 \\
\hline 1 & 11 & 44 & 19.80 & 14.24 \\
\hline 2 & 7 & 48 & 22.68 & 17.87 \\
\hline 2 & 8 & 11 & 0.00 & 0.00 \\
\hline 2 & 9 & 11 & 0.00 & 0.00 \\
\hline 2 & 10 & 39 & 16.32 & 11.02 \\
\hline 2 & 11 & 46 & 22.92 & 17.46 \\
\hline 3 & 7 & 25 & 0.00 & 0.00 \\
\hline 3 & 8 & 6 & 0.00 & 0.00 \\
\hline 3 & 9 & 11 & 0.00 & 0.00 \\
\hline 3 & 10 & 17 & 0.00 & 0.00 \\
\hline 3 & 11 & 27 & 6.56 & 1.58 \\
\hline 4 & 7 & 25 & 19.05 & 15.80 \\
\hline 4 & 8 & 49 & 35.51 & 28.19 \\
\hline 4 & 9 & 36 & 23.94 & 19.10 \\
\hline 4 & 10 & 21 & 1.39 & 0.00 \\
\hline 4 & 11 & 13 & 0.00 & 0.00 \\
\hline 5 & 7 & 5 & 0.00 & 0.00 \\
\hline 5 & 8 & 4 & 0.00 & 0.00 \\
\hline 5 & 9 & 45 & 35.31 & 30.79 \\
\hline 5 & 10 & 13 & 0.00 & 0.00 \\
\hline 5 & 11 & 15 & 0.00 & 0.00 \\
\hline 6 & 7 & 30 & 19.83 & 16.34 \\
\hline 6 & 8 & 2 & 0.00 & 0.00 \\
\hline 6 & 9 & 10 & 3.76 & 0.88 \\
\hline 6 & 10 & 10 & 0.00 & 0.00 \\
\hline 6 & 11 & 40 & 19.09 & 15.11 \\
\hline
\end{tabular}


Table 9: Link data and toll level solution

\begin{tabular}{|c|c|c|c|c|c|c|c|c|c|}
\hline \multirow{2}{*}{ From } & \multirow{2}{*}{ To } & \multicolumn{2}{|c|}{$\overline{\text { Link data }}$} & \multicolumn{2}{|c|}{ Link flows } & \multicolumn{4}{|c|}{ Toll levels } \\
\hline & & $t^{0}$ & $\zeta$ & $v^{0}$ & $v^{\mathrm{SO}}$ & $C=5$ & $C=20$ & $C=100$ & MSCP \\
\hline 1 & 18 & 0 & 0 & 57.64 & 41.61 & - & - & - & - \\
\hline 2 & 29 & 0 & 0 & 61.93 & 46.35 & - & - & - & - \\
\hline 3 & 31 & 0 & 0 & 6.56 & 1.58 & - & - & - & - \\
\hline 4 & 12 & 0 & 0 & 79.89 & 63.09 & - & - & - & - \\
\hline 5 & 14 & 0 & 0 & 35.31 & 30.79 & - & - & - & - \\
\hline 6 & 23 & 0 & 0 & 42.67 & 32.33 & - & - & - & - \\
\hline 12 & 13 & 6 & 2 & 73.72 & 59.34 & 10.29 & 10.15 & 16.88 & 9.92 \\
\hline 12 & 14 & 4 & 2 & 43.97 & 34.2 & - & - & - & 1.09 \\
\hline 13 & 7 & 0 & 0 & 77.24 & 60.88 & - & - & - & - \\
\hline 13 & 12 & 6 & 2 & 0 & 0 & - & - & - & - \\
\hline 13 & 17 & 5 & 1241 & 16.88 & 13.09 & 13.98 & 14.12 & - & 14.58 \\
\hline 14 & 12 & 4 & 2 & 37.8 & 30.44 & - & - & - & 0.69 \\
\hline 14 & 15 & 4 & 7 & 20.03 & 15.1 & - & - & - & 0.14 \\
\hline 14 & 23 & 4 & 2 & 61.61 & 49.89 & - & - & - & 4.95 \\
\hline 15 & 14 & 4 & 7 & 20.32 & 14.11 & - & 9.60 & - & 0.12 \\
\hline 15 & 16 & 2 & 3 & 18.64 & 15.1 & - & - & - & 0.06 \\
\hline 15 & 22 & 6 & 1550 & 1.39 & 0 & - & - & - & - \\
\hline 16 & 15 & 2 & 3 & 20.32 & 14.11 & - & - & - & 0.06 \\
\hline 16 & 17 & 4 & 1001 & 18.64 & 15.1 & 21.88 & 21.88 & 16.15 & 20.81 \\
\hline 16 & 20 & 5 & 75 & 0 & 0 & - & - & - & - \\
\hline 17 & 8 & 0 & 0 & 35.51 & 28.19 & - & - & - & - \\
\hline 17 & 13 & 5 & 1241 & 20.4 & 14.63 & - & - & - & 22.78 \\
\hline 17 & 16 & 4 & 1001 & 1.77 & 0.73 & - & - & - & 0.00 \\
\hline 17 & 19 & 2 & 521 & 0 & 0 & - & - & - & - \\
\hline 18 & 19 & 3 & 119 & 24.89 & 17.26 & 9.83 & - & - & 4.20 \\
\hline 18 & 29 & 2 & 1 & 39.6 & 30.74 & - & - & - & 0.36 \\
\hline 19 & 17 & 2 & 521 & 22.17 & 15.36 & 29.20 & 39.04 & 36.41 & 11.62 \\
\hline 19 & 18 & 3 & 119 & 0 & 0 & - & - & - & - \\
\hline 19 & 20 & 10 & 2306 & 10.39 & 7.82 & - & - & - & 3.46 \\
\hline 19 & 27 & 5 & 1157 & 0 & 0 & - & - & - & - \\
\hline 20 & 16 & 5 & 75 & 18.55 & 13.38 & - & - & - & 0.96 \\
\hline 20 & 19 & 10 & 2306 & 0 & 0 & - & - & - & - \\
\hline 20 & 21 & 3 & 12 & 0 & 0 & - & - & - & - \\
\hline 21 & 20 & 3 & 12 & 8.16 & 5.55 & - & - & - & - \\
\hline 21 & 22 & 5 & 75 & 14.25 & 10.46 & - & - & - & 0.36 \\
\hline 21 & 26 & 6 & 27 & 0 & 0 & - & - & - & - \\
\hline 21 & 27 & 4 & 1080 & 0 & 0 & - & - & - & - \\
\hline 21 & 28 & 8 & 1930 & 0 & 0 & - & - & - & - \\
\hline 22 & 15 & 6 & 1550 & 0 & 0 & - & - & - & - \\
\hline 22 & 21 & 5 & 75 & 0 & 0 & - & - & - & - \\
\hline 22 & 23 & 6 & 1550 & 14.25 & 10.46 & - & 10.04 & - & 7.42 \\
\hline 22 & 25 & 4 & 1061 & 1.39 & 2.04 & - & - & - & 0.00 \\
\hline 23 & 14 & 4 & 2 & 19.83 & 16.34 & - & - & - & 0.05 \\
\hline 23 & 22 & 6 & 1550 & 0 & 2.04 & - & - & - & 0.02 \\
\hline 23 & 24 & 3 & 1 & 98.7 & 74.3 & 18.92 & 17.45 & 24.59 & 12.19 \\
\hline 24 & 9 & 0 & 0 & 85.17 & 67.27 & - & - & - & - \\
\hline 24 & 23 & 3 & 1 & 0 & 0 & - & - & - & - \\
\hline 24 & 35 & 4 & 893 & 19.09 & 13.07 & - & 16.95 & - & 10.41 \\
\hline 25 & 22 & 4 & 1061 & 0 & 0 & - & - & - & - \\
\hline 25 & 26 & 5 & 1085 & 0 & 2.04 & - & - & - & 0.00 \\
\hline 25 & 34 & 4 & 1020 & 10.29 & 7.6 & - & - & - & 1.37 \\
\hline 26 & 21 & 6 & 27 & 0 & 0 & - & - & - & - \\
\hline
\end{tabular}


Table 9 - continued from previous page

\begin{tabular}{|c|c|c|c|c|c|c|c|c|c|}
\hline \multirow{2}{*}{ From } & \multirow{2}{*}{ To } & \multicolumn{2}{|c|}{ Link data } & \multicolumn{2}{|c|}{ Link flows } & \multicolumn{4}{|c|}{ Toll levels } \\
\hline & & $t^{0}$ & $\zeta$ & $v^{0}$ & $v^{\mathrm{SO}}$ & $C=5$ & $C=20$ & $C=100$ & MSCP \\
\hline 26 & 25 & 5 & 1085 & 8.9 & 7.6 & - & - & - & 1.45 \\
\hline 26 & 30 & 3 & 10 & 0 & 0 & - & - & - & - \\
\hline 26 & 33 & 3 & 53 & 21.34 & 15.87 & - & - & - & 1.35 \\
\hline 27 & 19 & 5 & 1157 & 7.67 & 5.93 & - & - & - & 0.57 \\
\hline 27 & 21 & 4 & 1080 & 17.9 & 12.46 & 5.81 & - & - & 10.43 \\
\hline 27 & 28 & 2 & 401 & 18.8 & 13.61 & - & - & - & 5.51 \\
\hline 27 & 29 & 3 & 3 & 0 & 0 & - & - & - & - \\
\hline 28 & 21 & 8 & 1930 & 4.51 & 3.55 & - & - & - & 0.12 \\
\hline 28 & 27 & 2 & 401 & 0 & 0 & - & - & - & - \\
\hline 28 & 30 & 2 & 554 & 14.29 & 10.06 & - & - & - & 2.27 \\
\hline 29 & 18 & 2 & 1 & 6.85 & 6.39 & - & - & - & 0.02 \\
\hline 29 & 27 & 3 & 3 & 44.37 & 31.99 & 7.50 & - & - & 1.25 \\
\hline 29 & 31 & 4 & 2 & 50.3 & 38.71 & - & - & - & 1.82 \\
\hline 30 & 26 & 3 & 10 & 30.24 & 21.43 & - & - & - & 0.84 \\
\hline 30 & 28 & 2 & 554 & 0 & 0 & - & - & - & - \\
\hline 30 & 31 & 4 & 958 & 0 & 0 & - & - & - & - \\
\hline 31 & 29 & 4 & 2 & 0 & 0 & - & - & - & - \\
\hline 31 & 30 & 4 & 958 & 15.95 & 11.38 & 7.74 & - & - & 6.41 \\
\hline 31 & 32 & 6 & 1373 & 22.45 & 15.99 & 36.91 & 36.37 & 36.46 & 35.83 \\
\hline 31 & 33 & 5 & 1130 & 18.46 & 12.93 & 11.96 & - & - & 12.59 \\
\hline 32 & 11 & 0 & 0 & 68.36 & 48.4 & - & - & - & - \\
\hline 32 & 31 & 6 & 1373 & 0 & 0 & - & - & - & - \\
\hline 32 & 33 & 2 & 401 & 0 & 0 & - & - & - & - \\
\hline 32 & 35 & 3 & 790 & 0 & 0 & - & - & - & - \\
\hline 33 & 26 & 3 & 53 & 0 & 0 & - & - & - & - \\
\hline 33 & 31 & 5 & 1130 & 0 & 0 & - & - & - & - \\
\hline 33 & 32 & 2 & 401 & 26.82 & 19.34 & 24.12 & 32.58 & 32.67 & 22.45 \\
\hline 33 & 34 & 4 & 960 & 12.98 & 9.46 & - & - & - & 3.07 \\
\hline 34 & 25 & 4 & 1020 & 0 & 0 & - & - & - & - \\
\hline 34 & 33 & 4 & 960 & 0 & 0 & - & - & - & - \\
\hline 34 & 35 & 2 & 451 & 23.27 & 17.06 & 20.68 & 29.06 & 27.10 & 15.29 \\
\hline 35 & 10 & 0 & 0 & 17.71 & 11.02 & - & - & - & - \\
\hline 35 & 24 & 4 & 893 & 5.56 & 6.04 & - & - & - & 0.47 \\
\hline 35 & 32 & 3 & 790 & 19.09 & 13.07 & 12.28 & - & - & 9.21 \\
\hline 35 & 34 & 2 & 451 & 0 & 0 & - & - & - & - \\
\hline Net b & nefit & & & & & 9323 & 9106 & 8501 & - \\
\hline Gross & enefit & & & & & 9393 & 9226 & 9201 & 9417 \\
\hline
\end{tabular}

